\title{
Some nano topological structures via ideals and graphs
}

\author{
Abd El-Fattah A. El-Atik ${ }^{1}$ (i) and Hanan Z. Hassan ${ }^{2^{*}}$ (D)
}

\author{
* Correspondence: H.pity88@yahoo. \\ com \\ ${ }^{2}$ Department of Mathematics, \\ Faculty of Science, Suez Canal \\ University, Ismailia, Egypt \\ Full list of author information is \\ available at the end of the article
}

\begin{abstract}
In this paper, new forms of nano continuous functions in terms of the notion of nano la-open sets called nano la-continuous functions, strongly nano la-continuous functions and nano la-irresolute functions will be introduced and studied. We establish new types of nano la-open functions, nano la-closed functions and nano la-homeomorphisms. A comparison between these types of functions and other forms of continuity will be discussed. We prove the isomorphism between simple graphs via the nano continuity between them. Finally, we apply these topological results on some models for medicine and physics which will be used to give a solution for some real-life problems.

Keywords: Ideals, Nano topology, Nla-open sets, Nla-continuous functions, Nlahomeomorphism functions, Directed graphs, Foetal circulation, Electric circuits

Mathematical Subject Classification 2010: 54C60, 54E55, 90D42, 03F55
\end{abstract}

\section{Introduction and preliminaries}

The theory of nano topology was introduced by Lellis Thivagar et al. [1]. They defined a nano topological space with respect to a subset $X$ of a universe $U$ which is defined based on lower and upper approximations of $X$.

Definition 1.1 [2]. Let $U$ be a certain set called the universe set and let $R$ be an equivalence relation on $U$. The pair $(U, R)$ is called an approximation space. Elements belonging to the same equivalence class are said to be indiscernible with one another. Let $X \subseteq U$.

(i) The lower approximation of $X$ with respect to $R$ is the set of all objects, which can be for certain classified as $X$ with respect to $R$ and it is denoted by $L_{R}(X)$. That is $L_{R}(X)=\underset{x \in U}{\cup}\left\{R_{x}: R_{x} \subseteq X\right\}$, where $R_{x}$ denotes to the equivalence class determined by $x$.

(ii) The upper approximation of $X$ with respect to $R$ is the set of all objects, which can be possibly classified as $X$ with respect to $R$ and it is denoted by $U_{R}(X)$. That is $U_{R}(X)=\underset{x \in U}{\cup}\left\{R_{x}: R_{x} \cap X \neq \varnothing\right\}$, where $R_{x}$ denotes to the equivalence class determined by $x$.

(c) The Author(s). 2020 Open Access This article is licensed under a Creative Commons Attribution 4.0 International License, which permits use, sharing, adaptation, distribution and reproduction in any medium or format, as long as you give appropriate credit to the original author(s) and the source, provide a link to the Creative Commons licence, and indicate if changes were made. The images or other third party material in this article are included in the article's Creative Commons licence, unless indicated otherwise in a credit line to the material. If material is not included in the article's Creative Commons licence and your intended use is not permitted by statutory regulation or exceeds the permitted use, you will need to obtain permission directly from the copyright holder. To view a copy of this licence, visit http://creativecommons.org/licenses/by/4.0/. 
(iii) The boundary region of $X$ with respect to $R$ is the set of all objects, which can be classified neither as $X$ nor as not $X$ with respect to $R$ and it is denoted by $B_{R}(X)$. That is $B_{R}(X)=U_{R}(X)-L_{R}(X)$, where $R_{x}$ denotes the equivalence class determined by $x$.

According to Pawlak's definition, $X$ is called a rough set if $U_{R}(X) \neq L_{R}(X)$.

Definition $1.2[3,4]$. Let $U$ be the universe and $R$ be an equivalence relation on $U$ and $\tau_{R}(X)=\left\{U, \varnothing, L_{R}(X), U_{R}(X), B_{R}(X)\right\}$, where $X \subseteq U$ and $\tau_{R}(X)$ satisfies the following axioms:

(i) $U$ and $\varnothing \in \tau_{R}(X)$;

(ii) The union of elements of any sub-collection of $\tau_{R}(X)$ is in $\tau_{R}(X)$;

(iii) The intersection of the elements of any finite sub-collection of $\tau_{R}(X)$ in $\tau_{R}(X)$.

That is $\tau_{R}(X)$ forms a topology on $U .\left(U, \tau_{R}(X)\right)$ is called a nano topological space. Nano-open sets are the elements of $\left(U, \tau_{R}(X)\right)$. It originates from the Greek word 'nanos' which means 'dwarf' in its modern scientific sense, an order to magnitude-one billionth. The topology is named as nano topology so because of its size since it has at most five elements [4]. The dual nano topology is $\left[\tau_{R}(X)\right]^{c}=F_{R}(X)$ and its elements are called nano closed sets.

Lellis Thivagar et al. [5] defined the concept of nano topological space via a direct simple graph.

Definition $1.3[5,6]$. A graph $G$ is an ordered pair of disjoint sets $(V, E)$, where $V$ is non-empty and $E$ is a subset of unordered pairs of $V$. The vertices and edges of a graph $G$ are the elements of $V=V(G)$ and $E=E(G)$, respectively. We say that a graph $G$ is finite (resp. infinite) if the set $V(G)$ is finite (resp. finite).

Definition 1.4 [5]. Let $G(V, E)$ be a directed graph and $u, v \in V(G)$, then:

(i) $u$ is invertex of $v$ if $\overrightarrow{u v} \in E(G)$.

(ii) $u$ is outvertex of $v$ if $\overrightarrow{v u} \in E(G)$.

(iii) The neighborhood of $v$ is denoted by $N(v)$, and given by $N(v)=\{v\} \cup\{u \in V(G)$ $: \overrightarrow{v u} \in E(G)\}$

Definition 1.5. Let $G(V, E)$ be a graph and $H$ be a subgraph of $G$. Then

(i) [5] The lower approximation $L: P(V(G)) \quad P(V(G))$ is $L_{N}(V(H))=\underset{v \in V(G)}{\cup}\{v: N(v)$ $\subseteq V(H)\}$

(ii) [7] The upper approximation $U: P(V(G)) \quad P(V(G))$ is $U_{N}(V(H))=\underset{v \in V(G)}{\cup}\{v: N(v)$ $\cap V(H) \neq \varnothing\}$;

(iii) [5] The boundary is $B_{N}(V(H))=U_{N}(V(H))-L_{N}(V(H))$.

Let $G$ be a graph, $N(v)$ be a neighbourhood of $v$ in $V$ and $H$ be a subgraph of $G$. $\tau_{N}(V(H))=\left\{V(G), \varnothing, L_{N}(V(H)), U_{N}(V(H)), B_{N}(V(H))\right\}$ forms a topology on $V(G)$ called the nano topology on $V(G)$ with respect to $V(H)$. $\left(V(G), \tau_{N}(V(H))\right)$ is a nano topological space induced by a graph $G$. 
Nano closure and nano interior of a set are also studied by Lellis Thivagar and Richard and put their definitions as:

Definition 1.6 [1]. If $\left(U, \tau_{R}(X)\right)$ is a nano topological space with respect to $X$ where $X \subseteq U$. If $A \subseteq U$, then the nano interior of $A$ is defined as the union of all nano-open subsets of $A$ and it is denoted by $\operatorname{NInt}(A)$. That is, $\operatorname{NInt}(A)$ is the largest nano-open subset of $A$. The nano closure of $A$ is defined as the intersection of all nano closed sets containing $A$ and it is denoted by $\operatorname{NCl}(A)$. That is, $\operatorname{NCl}(A)$ is the smallest nano closed set containing $A$.

Continuity of functions is one of the core concepts of topology. The notion of nano continuous functions was introduced by Lellis Thivagar and Richard [4]. They derived their characterizations in terms of nano closed sets, nano closure and nano interior. They also established nano-open maps, nano closed maps and nano homeomorphisms and their representations in terms of nano closure and nano interior.

Definition 1.7 [4]. Let $\left(U, \tau_{R}(X)\right)$ and $\left(V, \tau_{\hat{R}}(Y)\right)$ be nano topological spaces. Then a mapping $f:\left(U, \tau_{R}(X)\right) \rightarrow\left(V, \tau_{R}(Y)\right)$ is nano continuous on $U$ if the inverse image of every nano-open set in $V$ is nano-open in $U$.

Definition 1.8 [4]. A function $f:\left(U, \tau_{\hat{R}}(X)\right) \rightarrow\left(V, \tau_{\hat{R}}(Y)\right)$ is a nano-open map if the image of every nano-open set in $U$ is nano open in $V$. The mapping $f$ is said to be a nano closed map if the image of every nano closed set in $U$ is nano closed in $V$.

Definition 1.9 [4]. A function $f:\left(U, \tau_{\hat{R}}(X)\right) \rightarrow\left(V, \tau_{\hat{R}}(Y)\right)$ is said to be a nano homeomorphism if

(i) $f$ is $1-1$ and onto,

(ii) $f$ is nano continuous and

(iii) $f$ is nano open.

Graph isomorphism is a related task of deciding when two graphs with different specifications are structurally equivalent, that is whether they have the same pattern of connections. Nano homeomorphism between two nano topological spaces are said to be topologically equivalent. Here, we are formalizing the structural equivalence for the graphs and their corresponding nano topologies generated by them.

Definition 1.10 [8]. Two directed graphs $G$ and $H$ are isomorphic if there is an isomorphism $f$ between their underlying graphs that preserves the direction of each edge. That is, $e$ is directed from $u$ to $v$ if and only if $f(e)$ is directed from $f(u)$ to $f(v)$.

Definition 1.11 [8]. Two directed graphs $C$ and $D$ are isomorphic if $D$ can be obtained by relabeling the vertices of $C$, that is, if there is a bijection between the vertices of $C$ and those of $D$, such that the arcs joining each pair of vertices in $C$ agree in both number and direction with the arcs joining the corresponding pair of vertices in $D$.

The subject of ideals in topological spaces have been studied by Kuratowski [9] and Vaidyanathaswamy [10]. There have been many great attempts, so far, by topologies to use the concept of ideals for maneuvering investigations of different problems of topology. In this connection, one may refer to the works in [11-13].

Definition 1.12 [9]. An ideal $I$ on a set $X$ is a nonempty collection of subsets of $X$ which satisfies the conditions:

(i) $A \in I$ and $B \subseteq A$ implies $B \in I$, 
(ii) $A \in I$ and $B \in I$ implies $A \cup B \in I$.

(iii) The concept of a set operator ()$^{\alpha_{*}}: P(X) \rightarrow P(X)$ was introduced by Nasef [14] in 1992, which is called an $\alpha$-local function of $I$ with respect to $\tau$. In 2013, the notion of $I \alpha$-open set was introduced by Abd El-Monsef et al. [15] and has been studied by Radwan et al. [16, 17].

Definition 1.13 [15].: A subset $A$ of an ideal topological space $(X, \tau, I)$ is said to be $I \alpha$-open if it satisfies that $A \subseteq \operatorname{int}\left(c l^{\alpha \omega}[\operatorname{int}(A)]\right)$. The family of all Ia-open sets in ideal topological space $(X, \tau, I)$ is denoted by $I \alpha O(X)$.

It was made clear that each open set is $I \alpha$-open, but the converse may not be true, in general [16]. Radwan et al. have shown that the family of all $I \alpha$-open sets is a supra topology. In [18], the method of generating nano $I \alpha$-open sets are introduced and studied by Kozae et al.

Definition 1.14 [18]. A subset $X$ of a nano ideal topological space $(U, \tau(X), I)$ is said

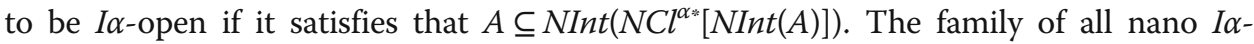
open sets in nano ideal topological space $(U, \tau(X), I)$ is denoted by $N I \alpha O(U)$. The elements of $[N I \alpha O(U)]^{c}$ are nano $I \alpha$-closed sets in nano ideal topological space $(U, \tau(X), I)$ and denoted by $N I \alpha C(U)$.

Also, discussions of various properties of nano $I \alpha$-open sets are given, such as nano $I \alpha$-closure and nano $I \alpha$-interior of a set.

Definition 1.15 [18]. Let $(U, \tau(X), I)$ be a nano ideal topological space and $A \subseteq U$. The nano $I \alpha$-interior of $A$ is defined as the union of all nano $I \alpha$-open subsets of $A$ and it is denoted by $N \operatorname{I\alpha }-\operatorname{Int}(A)$. That is, $N \operatorname{I} \alpha$-Int $(A)$ is the largest nano $\operatorname{I\alpha }$-open subset of $A$. The nano $I \alpha$-closure of $A$ is defined as the intersection of all nano $I \alpha$-closed sets containing $A$ and it is denoted by $\operatorname{NI} \alpha-C l(A)$. That is, $N I \alpha-C l(A)$ is the smallest nano $I \alpha-$ closed set containing $A$.

\section{Nla-continuous functions and Nla-homeomorphims}

We define some new functions in this section, say, nano $I \alpha$-continuous, nano $I \alpha$-open (closed), nano $I \alpha$-homeomorphism and other functions. Also, study the relationship between these functions, one to other and between them and nano continuous function, nano-open, nano closed and nano homeomorphism functions.

\subsection{New types of Nla-continuous functions:}

Definition 2.1.1. Let $f:\left(U, \tau_{R}(X), I\right) \rightarrow\left(V, \tau_{\hat{R}}(Y), J\right)$ be a function. $f$ is said to be

(i) Nano $I \alpha$-continuous function if $f^{-1}(B) \in N I \alpha O(U)$, for all $B \in \tau_{\tilde{R}}(Y)$.

(ii) Strongly nano $I \alpha$-continuous function if $f^{-1}(B) \in \tau_{R}(X)$, for all $B \in N I \alpha O(V)$.

(iii) Nano $I \alpha$ - irresolute continuous function if $f^{-1}(B) \in N I \alpha O(U)$ for all $B \in N I \alpha O(V)$.

Proposition 2.1.2. A function $f:\left(U, \tau_{R}(X), I\right) \rightarrow\left(V, \tau_{\hat{R}}(Y), J\right)$ is nano $I \alpha$-continuous function if and only if one of the following is satisfied;

(i) $f^{-1}(B) \in N I \alpha C(U)$, for all $B \in F_{\tilde{R}}(Y)$.

(ii) The inverse image of every member of the basis $B$ of $\tau_{\tilde{R}}(Y)$ is NI $\alpha$-open set in $U$. 
(iii) $N I \alpha-c l\left[f^{-1}(B)\right] \subseteq f^{-1}[N C l(B)]$, for all $B \subseteq V$.

(iv) $f^{-1}[N \operatorname{Int}(B)] \subseteq N I \alpha$-int $\left[f^{-1}(B)\right]$, for all $B \subseteq V$.

Proof:

(i) Necessity: let $f$ be nano $I \alpha$-continuous and $B \in F_{\tilde{R}}(Y)$. That is, $(V-B) \in \tau_{\tilde{R}}(Y)$. Since $f$ is nano $I \alpha$-continuous, $f^{-1}(V-B) \in N I \alpha O(U)$. That is, $\left(U-f^{-1}(B)\right) \in N I \alpha O(U)$. Therefore, $f^{-1}(B) \in N I \alpha C(U)$. Thus, the inverse image of every nano closed set in $V$ is NI $\alpha$-closed in $U$, if $f$ is nano I $\alpha$-continuous on $U$. Sufficiency: let $f^{-1}(B) \in N I \alpha C($ $U)$, for all $B \in F_{\tilde{R}}(Y)$. Let $B \in \tau_{\tilde{R}}(Y)$, then $(V-B) \in F_{\tilde{R}}(Y)$ and $f^{-1}(V-B) \in N I \alpha C(U)$. That is, $\left(U-f^{-1}(B)\right) \in N I \alpha C(U)$ and therefore $f^{-1}(B) \in N I \alpha O(U)$. Thus, the inverse image of every nano-open set in $V$ is NI $\alpha$-open in $U$. That is, $f$ is nano $I \alpha$-continuous on $U$.

(ii) Necessity: let $f$ be nano I $\alpha$-continuous on $U$. Let $B \in \dot{B}$. Then $B \in \tau_{\hat{R}}(Y)$. Since $f$ is nano I $\alpha$-continuous, $f^{-1}(B) \in N I \alpha O(U)$. That is, the inverse image of every member of $B$ is NIa-open set in $U$. Sufficiency: let the inverse image of every member of $\dot{B}$ be $N I \alpha$-open set in $U$. Let $G$ be a nano-open set in $V$. Then $G=\cup\left\{B: B \in B_{1}\right\}$, where $B_{1} \in \dot{B}$. Then $f^{-1}(G)=f^{-1}\left(\cup\left\{B: B \in B_{1}\right\}\right)=\cup\left\{f^{-1}(B): B \in B_{1}\right\}$, where each

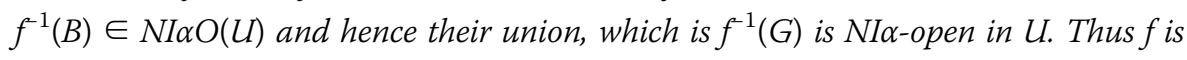
nano I $\alpha$-continuous on $U$.

(iii) Necessity: if $f$ is nano I $\alpha$-continuous and $B \subseteq V, N C l(B) \in F_{R(Y)}$ and from (i) $f^{-1}(N C l(B)) \in N I \alpha C(U)$. Therefore, NI $\alpha-c l\left(f^{-1}(N C l(B))\right)=f^{-1}(N C l(B))$. Since $B \subseteq$ $N C l(B), f^{-1}(B) \subseteq f^{-1}(N C l(B))$. Therefore, $N I \alpha-c l\left(f^{-1}(B)\right) \subseteq N I \alpha-c l\left(f^{-1}(N C l(B))\right)=$ $f^{-1}(N C l(B))$. That is, NI $\alpha-c l\left(f^{-1}(B)\right) \subseteq f^{-1}(N C l(B))$. Sufficiency: let $N I \alpha-c l\left(f^{-1}(B)\right) \subseteq$ $f^{-1}(N C l(B))$ for every $B \subseteq V$. Let $B \in F_{\tilde{R}}(Y)$, then $N C l(B)=B$. By assumption, NI $\alpha$ $c l\left(f^{-1}(B)\right) \subseteq f^{-1}(N C l(B))=f^{-1}(B)$. Thus, NI $\alpha-c l\left(f^{-1}(B)\right) \subseteq f^{-1}(B) . B u t f^{-1}(B) \subseteq N I \alpha-$ $c l\left(f^{-1}(B)\right)$. Therefore, NI $\alpha$-cl $\left(f^{-1}(B)\right)=f^{-1}(B)$. That is, $f^{-1}(B)$ is NI $\alpha$-closed in $U$ for every nano closed set $B$ in $V$. Therefore, $f$ is nano I $\alpha$-continuous on $U$.

(iv) Necessity: let $f$ be nano I $\alpha$-continuous and $B \subseteq V$. Then $\operatorname{NInt}(B) \in \tau_{\hat{R}}(Y)$. Therefore, $f^{-1}(\operatorname{NInt}(B)) \in N I \alpha O(U)$. That is, $f^{-1}(\operatorname{NInt}(B))=N I \alpha-\operatorname{int}\left(f^{-1}(\operatorname{NInt}(B))\right)$. Also, $N \operatorname{Int}(B) \subseteq B$ implies that NI $\alpha-i n t\left(f^{-1}(N \operatorname{Int}(B))\right) \subseteq N I \alpha-\operatorname{int}\left(f^{-1}(B)\right)$. Therefore $f^{-1}(N I n t(B))=N I \alpha-i n t\left(f^{-1}(N I n t(B))\right) \subseteq N I \alpha-i n t\left(f^{-1}(B)\right)$. That is, $f^{-1}(N \operatorname{Int}(B)) \subseteq N I \alpha-$ $\operatorname{int}\left(f^{-1}(B)\right)$. Sufficiency: let $f^{-1}(\operatorname{NInt}(B)) \subseteq N I \alpha-\operatorname{int}\left(f^{-1}(B)\right)$ for every subset $B$ of $V$. If $B$ $\in \tau_{\tilde{R}}(Y), B=\operatorname{NInt}(B)$. Also, $f^{-1}(B)=f^{-1}(\operatorname{NInt}(B))$, but $f^{-1}(\operatorname{NInt}(B)) \subseteq N I \alpha-i n t\left(f^{-1}(B)\right)$. That is, $f^{-1}(B)=f^{-1}(N I n t(B)) \subseteq N I \alpha$-int $\left(f^{-1}(B)\right)$. Therefore, $f^{-1}(B)=N I \alpha$-int $\left(f^{-1}(B)\right)$. Thus, $f^{-1}(B)$ is NIa-open in $U$ for every nano-open set $B$ in $V$. Therefore, $f$ is nano I $\alpha$-continuous.

Proposition 2.1.3. A function $f:\left(U, \tau_{R}(X), I\right) \rightarrow\left(V, \tau_{\hat{R}}(Y), J\right)$ is strongly nano $I \alpha-$ continuous function if and only if one of the following is satisfied;

(i) $f^{-1}(B) \in F_{R}(X)$, for all $B \in N I \alpha C(V)$.

(ii) The inverse image of every member of the basis $\dot{B}$ of $N I \alpha$-open set of $V$ is nanoopen set in $U$.

(iii) $N C l\left[f^{-1}(B)\right] \subseteq f^{-1}[N I \alpha-c l(B)]$, for all $B \subseteq V$. 
(iv) $f^{-1}[N I \alpha-\operatorname{int}(B)] \subseteq N \operatorname{Int}\left[f^{-1}(B)\right]$, for all $B \subseteq V$.

Proof:

(i) Necessity: let $f$ be strongly nano I $\alpha$-continuous and $B \in N I \alpha C(V)$. That is, $(V-$ $B) \in N I \alpha O(V)$, since $f$ is strongly nano I $\alpha$-continuous, $f^{-1}(V-B) \in \tau_{R}(X)$, and $(U-$ $\left.f^{-1}(B)\right) \in \tau_{R}(X)$. Therefore, $f^{-1}(B) \in F_{R}(X)$. Thus, $f^{-1}(B) \in F_{R}(X)$, for all $B \in$ $N I \alpha C(V)$, if $f$ is strongly nano I $\alpha$-continuous on $U$. Sufficiency: let $f^{-1}(B) \in F_{R}(X)$, for all $B \in N I \alpha C(V)$. Let $B \in N I \alpha O(V)$. Then $(V-B) \in N I \alpha C(V)$. Then, $f^{-1}(V-$ $B) \in F_{R}(X)$ that is, $\left(U-f^{-1}(B)\right) \in F_{R}(X)$. Therefore, $f^{-1}(B) \in \tau_{R}(X)$. Thus, the inverse image of every NI $\alpha$-open set in $V$ is nano-open in $U$. That is, $f$ is strongly nano I $\alpha$ continuous on $U$.

(ii) Necessity: let $f$ be strongly nano I $\alpha$-continuous on $U$. Let $B \in \dot{B}$. Then $B \in N I \alpha O(V)$. Since $f$ is strongly nano I $\alpha$-continuous, $f^{-1}(B) \in N I \alpha O(U)$. That is, the inverse image of every member of $B$ is nano-open set in $U$. Sufficiency: let the inverse image of every member of $B$ be nano-open set in $U$. Let $G$ be NI $\alpha$-open set in $V$. Then $G=\cup\left\{B: B \in B_{1}\right\}$, where $B_{1} \in B$. Then $f^{-1}(G)=f^{-1}\left(\cup\left\{B: B \in B_{1}\right\}\right)=\cup\left\{f^{-1}(B)\right.$ : $\left.B \in B_{1}\right\}$, where each $f^{-1}(B) \in \tau_{R}(X)$ and hence their union, which is $f^{-1}(G)$ is nanoopen in $U$. Thus $f$ is strongly nano I $\alpha$-continuous on $U$.

(iii) Necessity: if $f$ is strongly nano I $\alpha$-continuous and $B \subseteq V, N I \alpha-c l(B) \in N I \alpha C(V)$ and from (i) $f^{-1}(N I \alpha-c l(B)) \in F_{R}(X)$. Therefore, $N C l\left(f^{-1}(N I \alpha-c l(B))\right)=f^{-1}(N I \alpha-c l(B))$. Since $B \subseteq N I \alpha-c l(B), f^{-1}(B) \subseteq f^{-1}(N I \alpha-c l(B))$. Therefore, $N C l\left(f^{-1}(B)\right) \subseteq N C l\left(f^{-1}(N I \alpha-\right.$ $c l(B)))=\left(f^{-1}(N I \alpha-c l(B))\right)$. That is, $N C l\left(f^{-1}(B)\right) \subseteq\left(f^{-1}(N I \alpha-c l(B))\right)$. Sufficiency: let $N C l\left(f^{-1}(B)\right) \subseteq\left(f^{-1}(N I \alpha-c l(B))\right)$ for every $B \subseteq V$. Let $B \in N I \alpha C(V)$. Then $N I \alpha-c l(B)=$ $B$. By assumption, $N C l\left(f^{-1}(B)\right) \subseteq\left(f^{-1}(N I \alpha-c l(B))\right)=f^{-1}(B)$. Thus, $N C l\left(f^{-1}(B)\right) \subseteq$ $f^{-1}(B)$. But $f^{-1}(B) \subseteq N C l\left(f^{-1}(B)\right)$. Therefore, $N C l\left(f^{-1}(B)\right)=f^{-1}(B)$. That is, $f^{-1}(B) \in$ $F_{R}(X)$ for every NI $\alpha$-closed set $B$ in $V$. Therefore, $f$ is strongly nano I $\alpha$-continuous on $U$.

(iv) Necessity: let $f$ be strongly nano I $\alpha$-continuous and $B \subseteq V$. Then NI $\alpha$-int $(B) \in$ $N I \alpha O(V)$. Therefore, $\left(f^{-1}(N I \alpha-i n t(B))\right) \in \tau_{R}(X)$. That is, $f^{-1}(N I \alpha-i n t(B))=$

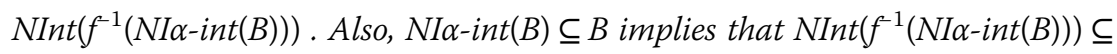
$\operatorname{NInt}\left(f^{-1}(B)\right)$. Therefore $f^{-1}(N I \alpha-\operatorname{int}(B))=\operatorname{NInt}\left(f^{-1}(N I \alpha-\operatorname{int}(B))\right) \subseteq \operatorname{NInt}\left(f^{-1}(B)\right)$. That is, $\left.f^{-1}(N I \alpha-\operatorname{int}(B))\right) \subseteq \operatorname{NInt}\left(f^{-1}(B)\right)$. Sufficiency: let $\left.f^{-1}(N I \alpha-\operatorname{int}(B))\right) \subseteq N \operatorname{Nint}\left(f^{-1}(B)\right)$ for every subset $B$ of $V$. If $B$ is NI $\alpha$-open set in $V, B=(N I \alpha-i n t(B))$. Also, $f^{-1}(B)=f^{-1}(N I \alpha-$ $\operatorname{int}(B))$, but $f^{-1}(N I \alpha-\operatorname{int}(B)) \subseteq \operatorname{NInt}\left(f^{-1}(B)\right)$. That is, $f^{-1}(B)=f^{-1}(N I \alpha-\operatorname{int}(B)) \subseteq$ $\operatorname{NInt}\left(f^{-1}(B)\right)$. Therefore, $f^{-1}(B)=\operatorname{NInt}\left(f^{-1}(B)\right)$. Thus, $f^{-1}(B)$ is nano-open in $U$ for every NI $\alpha$-open set $B$ in $V$. Therefore, $f$ is strongly nano I $\alpha$-continuous.

Proposition 2.1.4. A function $f:\left(U, \tau_{R}(X), I\right) \rightarrow\left(V, \tau_{\hat{R}}(Y), J\right)$ is nano $I \alpha$-irresolute continuous function if and only if one of the following is satisfied;

(i) $f^{-1}(B) \in N I \alpha C(U)$, for all $B \in N I \alpha C(V)$.

(ii) The inverse image of every member of the basis $B$ of $N I \alpha$-open set of $V$ is $N I \alpha$ open set in $U$.

(iii) $N I \alpha-c l\left[f^{-1}(B)\right] \subseteq f^{-1}[N I \alpha-c l(B)]$, for all $B \subseteq V$. 
(iv) $f^{-1}[N I \alpha-i n t(B)] \subseteq N I \alpha-i n t\left[f^{-1}(B)\right]$, for all $B \subseteq V$.

Proof:

(i) Necessity: let $f$ be nano $I \alpha$-irresolute continuous and $B \in N I \alpha C(V)$. That is, $(V-$ $B) \in N I \alpha O(V)$. Since $f$ is nano $I \alpha$-irresolute continuous, $f^{-1}(V-B) \in N I \alpha O(U)$. That is, $\left(U-f^{-1}(B)\right) \in N I \alpha O(U)$, and therefore $f^{-1}(B) \in N I \alpha C(U)$. Thus, $f^{-1}(B) \in$ $N I \alpha C(U)$, for all $B \in N I \alpha C(V)$, if $f$ is nano $I \alpha$-irresolute continuous on $U$. Sufficiency: let $f^{-1}(B) \in N I \alpha C(U)$, for all $B \in N I \alpha C(V)$. Let $B \in N I \alpha O(V)$. Then $(V$ $-B)$ is $I \alpha C(V)$. Then, $f^{-1}(V-B) \in I \alpha C(U)$, that is, $\left(U-f^{-1}(B)\right) \in I \alpha C(U)$. Therefore, $f^{-1}(B) \in N I \alpha O(U)$. Thus, $f^{-1}(B) \in N I \alpha O(U)$, for all $B \in N I \alpha O(V)$. That is, $f$ is nano $I \alpha$-irresolute continuous on $U$.

(ii) Necessity: let $f$ be nano I $\alpha$-irresolute continuous on $U$. Let $B \in \dot{B}$. Then $B \in$ $N I \alpha O(V)$. Since $f$ is nano I $\alpha$-irresolute continuous, $f^{-1}(B) \in N I \alpha O(U)$. That is, the inverse image of every member of $B$ is $N I \alpha O(U)$. Sufficiency: let the inverse image of every member of $B$ be NI $\alpha$-open set in $U$. Let $G \in N I \alpha O(V)$. Then $G=\cup\{B: B \in$ $\left.B_{1}\right\}$, where $B_{1} \in B$. Then $f^{-1}(G)=f^{-1}\left(\cup\left\{B: B \in B_{1}\right\}\right)=\cup\left\{f^{-1}(B): B \in B_{1}\right\}$, where each $f^{-1}(B) \in N I \alpha O(U)$ and hence their union, which is $f^{-1}(G)$. Thus $f$ is nano I $\alpha$ irresolute continuous on $U$.

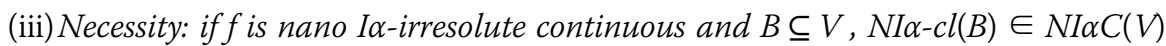
and from $(i) f^{-1}(N I \alpha-c l(B)) \in N I \alpha C(U)$. Therefore, NI $\alpha$-cl $\left(f^{-1}(N I \alpha-c l(B))\right)=f^{-1}(N I \alpha-$ cl $(B))$. Since $B \subseteq N I \alpha-c l(B), f^{-1}(B) \subseteq f^{-1}(N I \alpha-c l(B))$. Therefore, NI $\alpha-c l\left(f^{-1}(B)\right) \subseteq N I \alpha-$ $c l\left(f^{-1}(N I \alpha-c l(B))\right)=\left(f^{-1}(N I \alpha-c l(B))\right)$. That is, NI $\alpha-c l\left(f^{-1}(B)\right) \subseteq\left(f^{-1}(N I \alpha-c l(B))\right)$.

Sufficiency: let $N I \alpha-c l\left(f^{-1}(B)\right) \subseteq\left(f^{-1}(N I \alpha-c l(B))\right)$ for every $B \subseteq V$. Let $B \in N I \alpha C(V)$. Then NI $\alpha-c l(B)=B$. By assumption, NI $\alpha-c l\left(f^{-1}(B)\right) \subseteq\left(f^{-1}(N I \alpha-c l(B))\right)=f^{-1}(B)$. Thus, NI $\alpha-c l\left(f^{-1}(B)\right) \subseteq f^{-1}(B)$. But $f^{-1}(B) \subseteq N I \alpha-c l\left(f^{-1}(B)\right)$. Therefore, NI $\alpha$ $c l\left(f^{-1}(B)\right)=f^{-1}(B)$. That is, $f^{-1}(B)$ is NI $\alpha$-closed in $U$ for every NI $\alpha$-closed set $B$ in $V$. Therefore, $f$ is nano I $\alpha$-irresolute continuous on $U$.

(iv) Necessity: let $f$ be nano I $\alpha$-irresolute continuous and $B \subseteq V$. Then NI $\alpha$-int $(B) \in$ $N I \alpha O(V)$. Therefore, $\left(f^{-1}(N I \alpha-i n t(B))\right) \in N I \alpha O(U)$. That is, NI $\alpha$-int $\left(f^{-1}(N I \alpha-\right.$

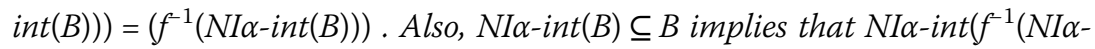
$\operatorname{int}(B))) \subseteq N I \alpha-\operatorname{int}\left(f^{-1}(B)\right)$. Therefore $f^{-1}(N I \alpha-\operatorname{int}(B))=N I \alpha-\operatorname{int}\left(f^{-1}(N I \alpha-\operatorname{int}(B))\right) \subseteq N I \alpha-$ $\operatorname{int}\left(f^{-1}(B)\right)$. That is, $\left.f^{-1}(N I \alpha-\operatorname{int}(B))\right) \subseteq N I \alpha-\operatorname{int}\left(f^{-1}(B)\right)$. Sufficiency: let $f^{-1}(N I \alpha-$ $\operatorname{int}(B))) \subseteq N I \alpha-\operatorname{int}\left(f^{-1}(B)\right)$ for every subset $B$ of $V$. If $B \in N I \alpha O(V), B=(N I \alpha-\operatorname{int}(B))$. Also, $f^{-1}(B)=f^{-1}(N I \alpha-\operatorname{int}(B))$ but, $f^{-1}(\operatorname{I\alpha }-\operatorname{int}(B)) \subseteq N I \alpha-\operatorname{int}\left(f^{-1}(B)\right)$. That is, $f^{-1}(B)=$ $f^{-1}(N I \alpha-i n t(B)) \subseteq N I \alpha-i n t\left(f^{-1}(B)\right)$. Therefore, $f^{-1}(B)=N I \alpha-\operatorname{int}\left(f^{-1}(B)\right)$. Thus, $f^{-1}(B)$ is

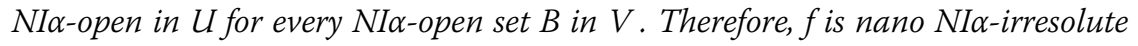
continuous.

Remark 2.1.5. The following implication shows the relationships between different types of nano continuous functions.

The converse of the above diagram is not reversible, in general, as shown in Example 2.1.6.

Example 2.1.6. Consider the nano ideal topological spaces $\left(U, \tau_{R}(X), I\right)$ and $\left(V, \tau_{\tilde{R}}(Y), J\right)$ such that $U=\{x, y, z\}, V=\{a, b, c\}, U / R=\{\{x\},\{y\},\{z\}\}, V / \tilde{R}=\{\{a\},\{b\},\{c\}\}$, if 
we take $X=\{x\}, Y=\{b\}$, then $\tau_{R}(X)=\{U, \varnothing,\{x\}\}, \tau_{\tilde{R}}(Y)=\{V, \varnothing,\{b\}\}$ and by taking $I=\{\varnothing,\{y\}\}, J=\{\varnothing,\{a\},\{c\},\{a, c\}\}$, so $N I \alpha O(U)=\{U, \varnothing,\{x\},\{x, y\},\{x, z\}\}, N I \alpha O(V)=\{V, \varnothing,\{b\},\{a$, $b\},\{b, c\}\}$. Define the function $f:\left(U, \tau_{R}(X), I\right) \rightarrow\left(V, \tau_{\hat{R}}(Y), J\right)$ such that

(i) $f(x)=f(y)=a, f(z)=c$. This function is nano $I \alpha$-continuous and nano continuous, but it is not nano $I \alpha$-irresolute continuous for $\{b, c\} \in N I \alpha O(V)$, but $f^{-1}(\{b, c\})=\{z\}$

$N I \alpha O(U)$. It is not strongly nano $I \alpha$-continuous since $\{a, b\} \in N I \alpha O(V)$, but $f^{-1}(\{a, b\})=\{x, y\} \notin \tau_{R}(X)$.

(ii) $f(x)=f(z)=b$ and $f(y)=a$. This function is nano $I \alpha$-irresolute continuous and nano $I \alpha$-continuous but neither strongly nano $I \alpha$-continuous nor nano continuous function for $\{b\} \in \tau_{\tilde{R}}(Y) \subseteq N I \alpha O(V)$, but $f^{-1}(\{b\})=\{x, z\} \quad \tau_{R}(X)$.

Remark 2.1.7. Consider the function $:\left(U, \tau_{R}(X), I\right) \rightarrow\left(V, \tau_{\hat{R}}(Y), J\right)$. The following statements are held.

(i) If $f$ is nano $I \alpha$-continuous function, it is not necessary that the $f(A) \in N I \alpha C(V)$, for all $A \in F_{\tilde{R}}(Y)$.

(ii) If $f$ is strongly nano $I \alpha$-continuous function, it is not necessary that $f(A) \in \tau_{\hat{R}}(Y)$, for all $A \in N I \alpha C(U)$.

(iii) If $f$ is nano $I \alpha$-irresolute continuous function, it is not necessary that $f(A) \in$ $N I \alpha C(V)$, for all $A \in N I \alpha C(U)$.

We show this remark by using the following example.

Example 2.1.8. Consider the nano ideal topological spaces $\left(U, \tau_{R}(X), I\right)$ and $\left(V, \tau_{\tilde{R}}(Y), J\right)$ such that $U=\{x, y, z\}, V=\{a, b, c\}, U / R=\{\{x\},\{y\},\{z\}\}, V / R=\{\{a\},\{b\},\{c\}\}$, if we take $X=\{x\}, Y=\{b\}$, then $\tau_{R}(X)=\{U, \varnothing,\{x\}\}, \tau_{\hat{R}}(Y)=\{V, \varnothing,\{b\}\}$ and by taking $I=\{\varnothing,\{y\}\}, J=\{\varnothing,\{a\},\{c\},\{a, c\}\}$, so $N I \alpha O(U)=\{U, \varnothing,\{x\},\{x, y\},\{x, z\}\}, N I \alpha O(V)=\{V, \varnothing,\{b\},\{a$, $b\},\{b, c\}\}$. Define the function $f:\left(U, \tau_{R}(X), I\right) \rightarrow\left(V, \tau_{\hat{R}}(Y), J\right)$ such that

(i) $f(x)=f(y)=f(z)=c$. This function is nano I $\alpha$-continuous. But $\{y, z\} \in F_{R}(X)$ and $f(\{y, z\})=\{c\} \notin N I \alpha C(V)$.

(ii) $f(x)=b$ and $f(y)=f(z)=c$. This function is strongly nano $I \alpha$-continuous. But $\{y\} \in$ $N I \alpha C(X)$ and $f(\{y\})=\{c\} \notin F_{\tilde{R}}(Y)$.

(iii) $f(x)=f(y)=c, f(z)=b$. This function is nano $I \alpha$-irresolute continuous. But $\{z\} \in$ $N I \alpha C(X)$ and $(\{z\})=\{b\} \notin N I \alpha C(V)$.

Definition 2.1.9. Let $f:\left(U, \tau_{R}(X), I\right) \rightarrow\left(V, \tau_{\hat{R}}(Y), J\right)$ be a function. $f$ is said to be

(i) Nano $I \alpha$-open [nano $I \alpha$-closed] function if $f(A) \in N I \alpha O(V)$, for all $A \in \tau_{R}(X)$ $[f(A) \in N I \alpha C(V)]$, for all $\left.A \in F_{R}(X)\right)$ respectively.

(ii) Strongly nano $I \alpha$-open [strongly nano $I \alpha$-closed] function if $f(A) \in \tau_{\tilde{R}}(Y)$, for all $A \in N I \alpha O(U)\left[f(A) \in F_{\tilde{R}}(Y)\right]$, for all $\left.A \in N I \alpha C(U)\right)$, respectively.

(iii) Nano $I \alpha$-almost open (nano $I \alpha$-almost closed) function if $f(A) \in N I \alpha O(V)$, for all $A \in N I \alpha O(U)[f(A) \in N I \alpha C(V)]$, for all $A \in N I \alpha C(U))$, respectively. 
Remark 2.1.10. The following implication shows the relationships between different types of nano-open functions.

The converse of the above diagram is not reversible, in general, as shown in Examples 2.1.11 and 2.1.12.

Example 2.1.11. Consider the nano ideal topological spaces $\left(U, \tau_{R}(X), I\right)$ and $\left(V, \tau_{\hat{R}}(\right.$ $Y), J)$ such that $U=\{x, y, z\}, V=\{a, b, c\}, U / R=\{\{x\},\{y\},\{z\}\}, V / \tilde{R}=\{\{a\},\{b\},\{$ $c\}\}$, if we take $X=\{x\}, Y=\{b\}$, then $\tau_{R}(X)=\{U, \varnothing,\{x\}\}, \tau_{\tilde{R}}(Y)=\{V, \varnothing,\{b\}\}$ and by taking $I=\{\varnothing,\{y\}\}, J=\{\varnothing,\{a\},\{b\},\{a, b\}\}$, so $N I \alpha O(U)=\{U, \varnothing,\{x\},\{x, y\},\{x, z\}\}, N I \alpha O(V)=$ $\{V, \varnothing,\{b\}\}$. Define the function $f:\left(U, \tau_{R}(X), I\right) \rightarrow\left(V, \tau_{\hat{R}}(Y), J\right)$ such that $f(x)=b$, $f(y)=a$ and $f(z)=c$. This function is nano $I \alpha$-open and nano-open, but it is neither nano $I \alpha$-almost open nor strongly nano $I \alpha$-open for $\{x, y\} \in N I \alpha O(U)$, but $f(\{x, y\})=\{a, b\} \notin$ $N I \alpha O(V)$ and $f(\{x, y\})=\{a, b\} \notin \tau_{\tilde{R}}(Y)$.

Example 2.1.12. Consider the nano ideal topological spaces $\left(U, \tau_{R}(X), I\right)$ and $\left(V, \tau_{\tilde{R}}(\right.$ $Y), J)$ such that $U=\{x, y, z\}, V=\{a, b, c\}, U / R=\{\{x\},\{y\},\{z\}\}, V / R=\{\{a\},\{b\},\{$ $c\}\}$, if we take $X=\{x\}, Y=\{b\}$, then $\tau_{R}(X)=\{U, \varnothing,\{x\}\}, \tau_{\hat{R}}(Y)=\{V, \varnothing,\{b\}\}$ and by taking $I=\{\varnothing,\{y\}\}, J=\{\varnothing,\{a\},\{c\},\{a, c\}\}$, so $N I \alpha O(U)=\{U, \varnothing,\{x\},\{x, y\},\{x, z\}\}, N I \alpha O(V)=$ $\{V, \varnothing,\{b\},\{a, b\},\{b, c\}\}$. Define the function $f:\left(U, \tau_{R}(X), I\right) \rightarrow\left(V, \tau_{\tilde{R}}(Y), J\right)$ such that $f(x)=b, f(y)=f(z)=a$. This function is nano $I \alpha$-open and nano $I \alpha$-almost open, but it is neither strongly nano $I \alpha$-open nor nano-open for $U \in \tau_{R}(X) \subseteq N I \alpha O(U)$, but $f(U)=\{a$, $b\} \notin \tau_{\hat{R}}(Y)$.

Remark 2.1.13. The following implication shows the relationships between different types of nano closed functions.

The converse of the above diagram is not reversible, in general, as shown in Examples2.1.14 and 2.1.15.

Example 2.1.14. Consider the nano ideal topological spaces $\left(U, \tau_{R}(X), I\right)$ and $\left(V, \tau_{\tilde{R}}(\right.$ $Y), J)$ such that $U=\{x, y, z\}, V=\{a, b, c\}, U / R=\{\{x\},\{y\},\{z\}\}, V / \tilde{R}=\{\{a\},\{b\},\{$ $c\}\}$, if we take $X=\{x\}, Y=\{b\}$, then $\tau_{R}(X)=\{U, \varnothing,\{x\}\}, \tau_{\tilde{R}}(Y)=\{V, \varnothing,\{b\}\}$ and by taking $I=\{\varnothing,\{y\}\}, J=\{\varnothing,\{a\},\{b\},\{a, b\}\}$, so $N I \alpha O(U)=\{U, \varnothing,\{x\},\{x, y\},\{x, z\}\}, N I \alpha O(V)=$ $\{V, \varnothing,\{b\}\}$. Define the function $f:\left(U, \tau_{R}(X), I\right) \rightarrow\left(V, \tau_{\tilde{R}}(Y), J\right)$ such that $f(x)=f(y)=$ $a$ and $f(z)=c$. This function is nano $I \alpha$-closed and nano closed, but it is neither nano $I \alpha$-almost closed nor strongly nano I $\alpha$-closed for $\{y\} \in N I \alpha C(U)$, but $f(\{y\})=\{a\} \notin$ $N I \alpha C(V)$ and $f(\{y\})=\{a\} \notin F_{\hat{R}}(Y)$.

Example 2.1.15. Consider the nano ideal topological spaces $\left(U, \tau_{R}(X), I\right)$ and $\left(V, \tau_{\hat{R}}(Y)\right.$, $J$ ) such that $U=\{x, y, z\}, V=\{a, b, c\}, U / R=\{\{x\},\{y\},\{z\}\}, V / R=\{\{a\},\{b\},\{c\}\}$, if we take $X=\{x\}, Y=\{b\}$, then $\tau_{R}(X)=\{U, \varnothing,\{x\}\}, \tau_{\tilde{R}}(Y)=\{V, \varnothing,\{b\}\}$ and by taking $I=\{\varnothing,\{y\}\}, J=\{\varnothing,\{a\},\{c\},\{a, c\}\}$, so $N I \alpha O(U)=\{U, \varnothing,\{x\},\{x, y\},\{x, z\}\}, N I \alpha O(V)=\{V, \varnothing,\{b\}$, $\{a, b\},\{b, c\}\}$. Define the function $f:\left(U, \tau_{R}(X), I\right) \rightarrow\left(V, \tau_{\tilde{R}}(Y), J\right)$ such that $f(x)=a, f(y)=$ $f(z)=c$. This function is nano $I \alpha$-closed and nano $I \alpha$-almost closed, but it is neither strongly nano $I \alpha$-closed nor nano closed for $\{y, z\} \in F_{R}(X) \subseteq N I \alpha C(U)$ but, $f(\{y, z\})=\{c\} \notin F_{\tilde{R}}(Y)$.

\section{Nla-homeomorphism functions:}

Definition 2.2.1. Let $f:\left(U, \tau_{R}(X), I\right) \rightarrow\left(V, \tau_{\tilde{R}}(Y), J\right)$ be a bijective function. $\quad$ is said to be 
(i) Nano $I \alpha$-homeomorphism function if $f$ and $f^{-1}$ are both nano $I \alpha$-continuous functions.

(ii) Strongly nano $I \alpha$-homeomorphism function if $f$ and $f^{-1}$ are both strongly nano $I \alpha$ continuous functions.

(iii) Nano $I \alpha$-irresolute homeomorphism function if $f$ and $f^{-1}$ are both nano $I \alpha$ irresolute continuous functions.

Remark 2.2.2. Let $f:\left(U, \tau_{R}(X), I\right) \rightarrow\left(V, \tau_{\tilde{R}}(Y), J\right)$ be a bijective function. $f$ is said to be

(i) Nano $I \alpha$-homeomorphism function if $f$ is both nano $I \alpha$-continuous and nano $I \alpha$ open function.

(ii) Strongly nano $I \alpha$-homeomorphism function if $f$ is both strongly nano $I \alpha$ continuous and is strongly nano $I \alpha$-open function.

(iii) Nano $I \alpha$-irresolute homeomorphism function if $f$ is both nano $I \alpha$-irresolute continuous and nano $I \alpha$-almost open function.

Proposition 2.2.3. Let $f:\left(U, \tau_{R}(X), I\right) \rightarrow\left(V, \tau_{\tilde{R}}(Y), J\right)$ and $g:\left(V, \tau_{\tilde{R}}(Y), J\right) \rightarrow(W$, $\left.\tau_{\tilde{R}}(Z), K\right)$ be two functions. Then $g \circ f$ is

(i) Nano continuous function if $f, g$ are strongly nano $I \alpha$-continuous and nano $I \alpha$ continuous functions.

(ii) Nano $I \alpha$-continuous function if $f, g$ are nano $I \alpha$-irresolute continuous and nano continuous functions.

(iii) Strongly nano $I \alpha$-continuous function if $f, g$ are strongly nano $I \alpha$-continuous and nano $I \alpha$-irresolute continuous functions.

Proof:

(i) Take $C \subseteq W$ such that $C \in \tau_{\tilde{R}}(Z)$, then $g^{-1}(C) \in N I \alpha O(V)$ and $f^{-1}\left(g^{-1}(C)\right) \in \tau_{R}(X)$. Thus $C \in \tau_{\hat{R}}(Z),(g \circ f)^{-1} \in \tau_{R}(X)$, so $g \circ f$ is nano continuous function.

(ii) Take $C \subseteq W$ such that $C \in \tau_{\tilde{R}}(Z)$, then $g^{-1}(C) \in \tau_{\tilde{R}}(Y) \subseteq N I \alpha O(V)$ and $f^{-1}\left(g^{-1}(C)\right) \in$ $N I \alpha O(U)$. Thus $C \in \tau_{\tilde{R}}(Z),(g \circ f)^{-1} \in N I \alpha O(U)$, so $g \circ f$ is nano $I \alpha$-continuous function.

(iii) Take $C \subseteq W$ such that $C \in N I \alpha O(W)$, then $g^{-1}(C) \in N I \alpha O(V)$ and $f^{-1}\left(g^{-1}(C)\right) \in \tau_{R}(X)$. Thus $C \in N I \alpha O(W),(g \circ f)^{-1} \in \tau_{R}(X)$, and $g \circ f$ is strongly nano $I \alpha$-continuous function.

Proposition 2.2.4. Let $f:\left(U, \tau_{R}(X), I\right) \rightarrow\left(V, \tau_{\tilde{R}}(Y), J\right)$ and $g:\left(V, \tau_{\tilde{R}}(Y), J\right) \rightarrow(W$, $\left.\tau_{\hat{R}}(Z), K\right)$ be two functions. Then $g \circ f$ is nano $I \alpha$-irresolute continuous function in the following cases.

(i) If $f, g$ are both nano $I \alpha$-irresolute continuous functions.

(ii) If $f, g$ are nano $I \alpha$-irresolute continuous and strongly nano $I \alpha$-continuous functions, respectively. 
(iii) If $f, g$ are nano $I \alpha$-continuous and strongly nano $I \alpha$-continuous functions, respectively.

Proof: Take $C \subseteq W$ such that $C \in I \alpha O(W)$.

(i) Since $C \in N I \alpha O(W)$ then $g^{-1}(C) \in N I \alpha O(V)$ and $f^{-1}\left(g^{-1}(C)\right) \in N I \alpha O(U)$.

(ii) Since $C \in N I \alpha O(W)$ then $g^{-1}(C) \in \tau_{\tilde{R}}(Y) \subseteq N I \alpha O(Y)$ and $f^{-1}\left(g^{-1}(C)\right) \in N I \alpha O(U)$.

(iii) Since $C \in N I \alpha O(W)$ then $g^{-1}(C) \in \tau_{\hat{R}}(Y)$ and $f^{-1}\left(g^{-1}(C)\right) \in N I \alpha O(U)$.

Thus, we have that $C \in N I \alpha O(W),(g \circ f)^{-1} \in N I \alpha O(U)$, and $g \circ f$ is nano $I \alpha$-irresolute continuous function.

Proposition 2.2.5. Let $f:\left(U, \tau_{R}(X), I\right) \rightarrow\left(V, \tau_{\hat{R}}(Y), J\right)$ and $g:\left(V, \tau_{\hat{R}}(Y), J\right) \rightarrow(W$, $\left.\tau_{\hat{R}}(Z), K\right)$ be two functions. Then $g \circ f$ is nano-open function in the following cases:

(i) If $f, g$ are nano $I \alpha$-open and strongly nano $I \alpha$-open functions, respectively.

(ii) If $f, g$ are nano-open and strongly nano $I \alpha$-open functions, respectively.

Proof: Take $A \subseteq U$ such that $A \in \tau_{R}(X)$.

(i) Since $A \in \tau_{R}(X)$ then $f(A) \in \tau_{\tilde{R}}(Y) \subseteq N I \alpha O(V)$ and $g(f(A)) \in \tau_{\tilde{R}}(Z)$.

(ii) Since $A \in \tau_{R}(X)$ then $f(A) \in \tau_{\hat{R}}(Y)$ and $g(f(A)) \in \tau_{\hat{R}}(Z)$.

Thus, in each case, we have that $A \in \tau_{R}(X),(g \circ f) \in \tau_{\hat{R}}(Z)$, and $g \circ f$ is nano-open function.

Proposition 2.2.6. Let $f:\left(U, \tau_{R}(X), I\right) \rightarrow\left(V, \tau_{\tilde{R}}(Y), J\right)$ and $g:\left(V, \tau_{\tilde{R}}(Y), J\right) \rightarrow(W$, $\left.\tau_{\hat{R}}(Z), K\right)$ be two functions. Then $g \circ f$ is nano $I \alpha$-open function in the following cases:

(i) If $f, g$ are nano $I \alpha$-open and nano $I \alpha$-almost open functions, respectively.

(ii) If $f, g$ are nano-open and nano $I \alpha$-almost open functions, respectively.

(iii) If $f, g$ are nano-open and nano $I \alpha$-open functions, respectively.

Proof: Take $A \subseteq U$ such that $A \in \tau_{R}(X)$.

(i) Since $A \in \tau_{R}(X)$ then $f(A) \in N I \alpha O(V)$ and $g(f(A)) \in N I \alpha O(W)$.

(ii) Since $A \in \tau_{R}(X)$ then $f(A) \in \tau_{\hat{R}}(Y) \subseteq N I \alpha O(V)$ and $g(f(A)) \in N I \alpha O(W)$.

(iii) Since $A \in \tau_{R}(X)$ then $f(A) \in \tau_{\hat{R}}(Y)$ and $g(f(A)) \in N I \alpha O(W)$.

Thus, in each case, we have that $A \in \tau_{R}(X),(g \circ f) \in N I \alpha O(W)$, and $g \circ f$ is nano $I \alpha$-open function.

Proposition 2.2.7. Let $f:\left(U, \tau_{R}(X), I\right) \rightarrow\left(V, \tau_{\hat{R}}(Y), J\right)$ and $g:\left(V, \tau_{\hat{R}}(Y), J\right) \rightarrow(W$, $\left.\tau_{\hat{R}}(Z), K\right)$ be two functions. Then $g \circ f$ is strongly nano $I \alpha$-open function in the following cases.

(i) If $f, g$ are nano $I \alpha$-almost open and strongly nano $I \alpha$-open functions, respectively.

(ii) If $f, g$ are both strongly nano $I \alpha$-open functions.

(iii) If $f, g$ are strongly nano $I \alpha$-open and nano-open functions, respectively. 
Proof: Take $A \subseteq U$ such that $A \in N I \alpha O(U)$.

(i) Since $A \in N I \alpha O(U)$ then $f(A) \in N I \alpha O(V)$ and $g(f(A)) \in \tau_{\tilde{R}}(Z)$.

(ii) Since $A \in N I \alpha O(U)$ then $f(A) \in \tau_{\tilde{R}}(Y) \subseteq N I \alpha O(V)$ and $g(f(A)) \in \tau_{\tilde{R}}(Z)$.

(iii) Since $A \in N I \alpha O(U)$ then $f(A) \in \tau_{\tilde{R}}(Y)$ and $g(f(A)) \in \tau_{\tilde{R}}(Z)$.

Thus, we have that $A \in N I \alpha O(U),(g \circ f) \in \tau_{\tilde{R}}(Z)$, and $g \circ f$ is strongly nano $I \alpha$-open function.

Proposition 2.3.6. Let $f:\left(U, \tau_{R}(X), I\right) \rightarrow\left(V, \tau_{\tilde{R}}(Y), J\right)$ and $g:\left(V, \tau_{\tilde{R}}(Y), J\right) \rightarrow(W$, $\left.\tau_{\hat{R}}(Z), K\right)$ be two functions. Then $g \circ f$ is nano $I \alpha$-almost open function in the following cases:

(i) If $f, g$ are both nano $I \alpha$-almost open functions.

(ii) If $f, g$ are strongly nano $I \alpha$-open and nano $I \alpha$-almost open functions, respectively.

(iii) If $f, g$ are strongly nano $I \alpha$-open and nano $I \alpha$-open functions, respectively.

Proof: Take $A \subseteq U$ such that $A \in N I \alpha O(U)$.

(i) Since $A \in N I \alpha O(U)$ then $f(A) \in N I \alpha O(V)$ and $g(f(A)) \in N I \alpha O(W)$.

(ii) Since $A \in N I \alpha O(U)$ then $f(A) \in \tau_{\hat{R}}(Y) \subseteq N I \alpha O(V)$ and $g(f(A)) \in N I \alpha O(W)$.

(iii) Since $A \in N I \alpha O(U)$ then $f(A) \in \tau_{\tilde{R}}(Y)$ and $g(f(A)) \in N I \alpha O(W)$.

Thus, we have that $A \in N I \alpha O(U),(g \circ f) \in N I \alpha O(W)$, and $g \circ f$ is nano $I \alpha$-almost open function.

\section{Ideal expansion on topological rough sets and topological graphs}

We extend both the rough sets and graphs induced by topology in Examples 3.1 and 3.2 respectively. The expansion will be used to give a decision for some diseases as flu.

Example 3.1. An example of a decision table is presented in Table 1. Four attributes [temperature, headache, nausea and cough], one decision [flu] and six cases.

Let

(i) $R_{1}=\{$ Temperature $\}$, the family of all equivalence classes of $I N D(R)$ is $U / R_{1}=\{\{1$, $3,4\},\{2\},\{5,6\}\}$

(ii) $R_{2}=\{$ Temperature, Headache $\}$, then $U / R_{2}=\{\{1,4\},\{2\},\{3\},\{5\},\{6\}\}$

(iii) $R_{3}=\{$ Headache, Cough $\}$, then $U / R_{3}=\{\{1,4\},\{2,5\},\{3\},\{6\}\}$.

If we take, $X=\left\{x:[x]_{\text {Nausea }}=\right.$ no $\}=\{1,3,5\}$ then

(i) $L_{R_{1}}(X)=\emptyset, U_{R_{1}}(X)=\{1,3,4,5,6\}$ and $B_{R_{1}}(X)=\{1,3,4,5,6\}$. Thus $\tau_{R_{1}}(X)=\{$ $U, \emptyset,\{1,3,4,5,6\}\}$.

(ii) $L_{R_{2}}(X)=\{3,5\}, U_{R_{2}}(X)=\{1,3,4,5\}$, and $B_{R_{2}}(X)=\{1,4\}$. Thus $\tau_{R_{2}}(X)=\{X$, $\emptyset,\{1,4\},\{3,5\},\{1,3,4,5\}\}$.

(iii) $L_{R_{3}}(X)=\{3\}, U_{R_{3}}(X)=\{1,2,3,4,5\}$ and $B_{R_{3}}(X)=\{1,2,4,5\}$. Thus $\tau_{R_{3}}(X)=\{$ $X, \emptyset,\{3\},\{1,2,4,5\},\{1,2,3,4,5\}\}$. 
Table 1 A decision table for flu

\begin{tabular}{llllll}
\hline Case & Attributes & & & \multicolumn{2}{c}{ Decision } \\
\cline { 2 - 5 } & Temperature & Headache & Nausea & Cough & Flu \\
\hline 1 & High & Yes & No & Yes & Yes \\
2 & Very high & Yes & Yes & No & Yes \\
3 & High & No & No & No & No \\
4 & High & Yes & Yes & Yes & Yes \\
5 & Normal & Yes & No & No & No \\
6 & Normal & No & Yes & Yes & No \\
\hline
\end{tabular}

If we take, $I=\{\varnothing,\{2\},\{4\},\{2,4\}\}$ then

(i) $(N I \alpha O(U))_{1}=\{U, \varnothing,\{1,3,4,5,6\}\}$.

(ii) $(N I \alpha O(U))_{2}=\{U, \varnothing,\{1,4\},\{3,5\},\{1,3,4,5\},\{1,2,3,4,5\},\{1,3,4,5,6\}\}$.

(iii) $(N I \alpha O(U))_{3}=\{U, \varnothing,\{3\},\{1,2,4,5\},\{1,2,3,4,5\}\}$.

Define a function $f:\left(U, \tau_{R_{3}}(X), I\right) \rightarrow\left(V, \tau_{R_{2}}(X), I\right)$ such that $f(1)=1, f(2)=4, f(3)=$ 2, $f(4)=1, f(5)=4$ and $f(6)=6$. This function is nano $I \alpha$-continuous and nano continuous, but it is neither nano $I \alpha$ - irresolute continuous nor strongly nano $I \alpha$-continuous for, $\{1,3,4,5,6\} \in(N I \alpha O(U))_{2}$, but $f^{-1}(\{1,3,4,5,6\})=\{1,2,4,5,6\} \notin(N I \alpha O(U))_{3}$.

Define a function $f:\left(U, \tau_{R_{2}}(X), I\right) \rightarrow\left(V, \tau_{R_{3}}(X), I\right)$ such that $f(1)=1, f(2)=6, f(3)=$ $2, f(4)=4, f(5)=5$ and $f(6)=2$. This function is nano I $\alpha$-continuous and nano I $\alpha$ - irresolute continuous, but it is neither nano continuous nor strongly nano $I \alpha$-continuous for, $\{1,2,4,5\} \in \tau_{R_{3}}(X) \subseteq(N I \alpha O(U))_{3}$, but $f^{-1}(\{1,2,4,5\})=\{1,3,4,5,6\} \notin \tau_{R_{2}}(X)$.

Example 3.2. A nano topology will be induced by a general graph. Figure 1 shows two different simple directed graphs $G$ and $H$, where $V(G)=\left\{v_{1}, v_{2}, v_{3}, v_{4}, v_{5}, v_{6}\right\}$ and $V(H)=\left\{w_{1}, w_{2}, w_{3}, w_{4}, w_{5}, w_{6}\right\}$.

From the previous figure $N\left(v_{1}\right)=\left\{v_{1}, v_{2}, v_{4}, v_{5}\right\}, N\left(v_{2}\right)=\left\{v_{2}, v_{3}, v_{6}\right\}, N\left(v_{3}\right)=\left\{v_{3}, v_{4}, v_{5}\right\}$, $N\left(v_{4}\right)=\left\{v_{4}, v_{6}\right\}, N\left(v_{5}\right)=\left\{v_{5}\right\}$ and $N\left(v_{6}\right)=\left\{v_{5}, v_{6}\right\}$. Let $X=\left\{v_{5}\right\}$, then $L(X)=\left\{v_{5}\right\}, U(X)=\left\{v_{1}\right.$, $\left.v_{3}, v_{5}, v_{6}\right\}$ and $b(X)=\left\{v_{1}, v_{3}, v_{6}\right\}$, which mean that $\tau_{R}=\left\{V(G), \varnothing,\left\{v_{5}\right\},\left\{v_{1}, v_{3}, v_{6}\right\},\left\{v_{1}, v_{3}\right.\right.$,
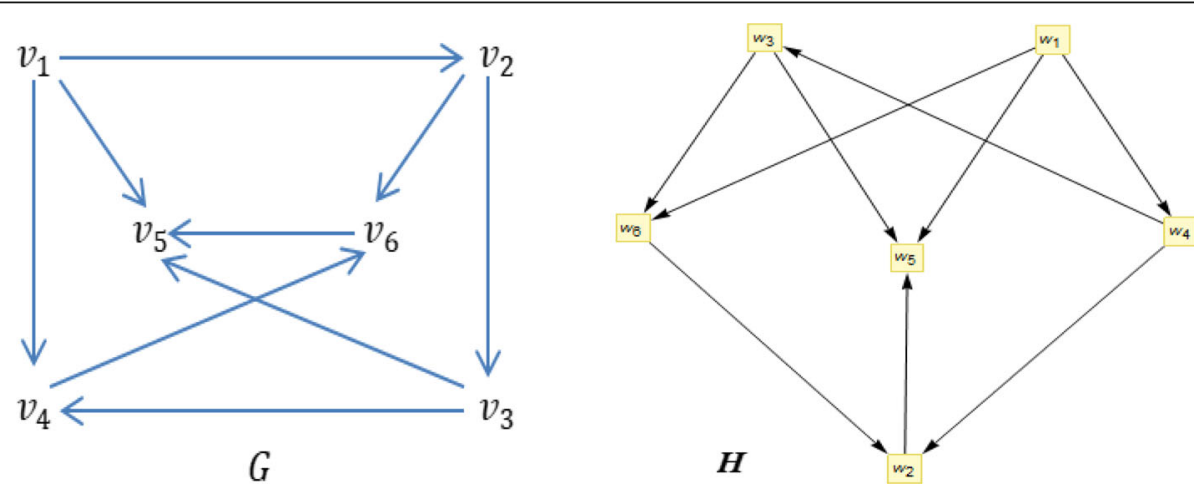

Fig. 1 Simple directed graphs 
$\left.\left.v_{5}, v_{6}\right\}\right\}$. take $I=\left\{\varnothing,\left\{v_{1}\right\}\right\}$ then $N I \alpha O(V(G))=\left\{V(G), \varnothing,\left\{v_{5}\right\},\left\{v_{1}, v_{3}, v_{6}\right\},\left\{v_{1}, v_{3}, v_{5}, v_{6}\right\},\left\{v_{1}\right.\right.$, $\left.\left.v_{2}, v_{3}, v_{5}, v_{6}\right\},\left\{v_{1}, v_{3}, v_{4}, v_{5}, v_{6}\right\}\right\}$.

Similarly $N\left(w_{1}\right)=\left\{w_{1}, w_{4}, w_{5}, w_{6}\right\}, N\left(w_{2}\right)=\left\{w_{2}, w_{5}\right\}, N\left(w_{3}\right)=\left\{w_{3}, w_{5}, w_{6}\right\}, N\left(w_{4}\right)=\left\{w_{2}, w_{3}, w_{4}\right\}$, $N\left(w_{5}\right)=\left\{w_{5}\right\}$ and $N\left(w_{6}\right)=\left\{w_{2}, w_{6}\right\}$. Let $Y=\left\{w_{5}\right\}$, then $L(Y)=\left\{w_{5}\right\}, U(Y)=\left\{w_{1}, w_{2}, w_{3}\right.$, $\left.w_{5}\right\}$ and $b(Y)=\left\{w_{1}, w_{2}, w_{3}\right\}$, which mean that $\tau_{\hat{R}}=\left\{V(H), \varnothing,\left\{w_{5}\right\},\left\{w_{1}, w_{2}, w_{3}\right\},\left\{w_{1}, w_{2}\right.\right.$ $\left.\left., w_{3}, w_{5}\right\}\right\}$. Take $J=\left\{\varnothing,\left\{w_{1}\right\}\right\}$ then $N I \alpha O(V(H))=\left\{V(H), \varnothing,\left\{w_{5}\right\},\left\{w_{1}, w_{2}, w_{3}\right\},\left\{w_{1}, w_{2}\right.\right.$, $\left.\left.w_{3}, w_{5}\right\},\left\{w_{1}, w_{2}, w_{3}, w_{4}, w_{5}\right\},\left\{w_{1}, w_{2}, w_{3}, w_{5}, w_{6}\right\}\right\}$.

Define a function $f:\left(V(G), \tau_{R}(X), I\right) \rightarrow\left(V(H), \tau_{\hat{R}}(Y), J\right)$ such that $f\left(v_{1}\right)=w_{1}$, $f\left(v_{2}\right)=w_{4}, f\left(v_{3}\right)=w_{3}, f\left(v_{4}\right)=w_{6}, f\left(v_{5}\right)=w_{5}$ and $f\left(v_{6}\right)=w_{6}$. This function is nano continuous, nano $I \alpha$-continuous and nano $I \alpha$ - irresolute, but it is not strongly nano $I \alpha$-continuous for, $\left\{w_{1}, w_{2}, w_{3}, w_{4}, w_{5}\right\} \in N I \alpha O(V(H))$ but $f^{-1}\left(\left\{w_{1}, w_{2}, w_{3}, w_{4}, w_{5}\right\}\right)=\left\{v_{1}\right.$, $\left.v_{2}, v_{3}, v_{5}, v_{6}\right\} \notin \tau_{R}(X)$, and this function is nano open, nano $I \alpha$-open and nano $I \alpha$-almost open, but it is not strongly nano $I \alpha$-open for, $\left\{v_{1}, v_{3}, v_{4}, v_{5}, v_{6}\right\} \in N I \alpha O(V(G))$, but $f\left(\left\{v_{1}, v_{3}, v_{4}, v_{5}, v_{6}\right\}\right)=\left\{w_{1}, w_{2}, w_{3}, w_{5}, w_{6}\right\} \notin \tau_{\hat{R}}(Y)$, also this function is one to one and onto, therefore it is nano homeomorphism, nano $I \alpha$-homeomorphism and nano $I \alpha$-irresolute homeomorphism, but it is not strongly nano $I \alpha$ homeomorphism.

\section{Topological models in terms of graphs and nano topology}

In this section, we apply these new types of functions on some real-life problems, especially, in medicine and physics.

\subsection{The foetal circulation}

In this section, we apply some of the graphs, nano topology and NI $\alpha$-open sets on some of the medical application such as the blood circulation in the foetus. [D1,

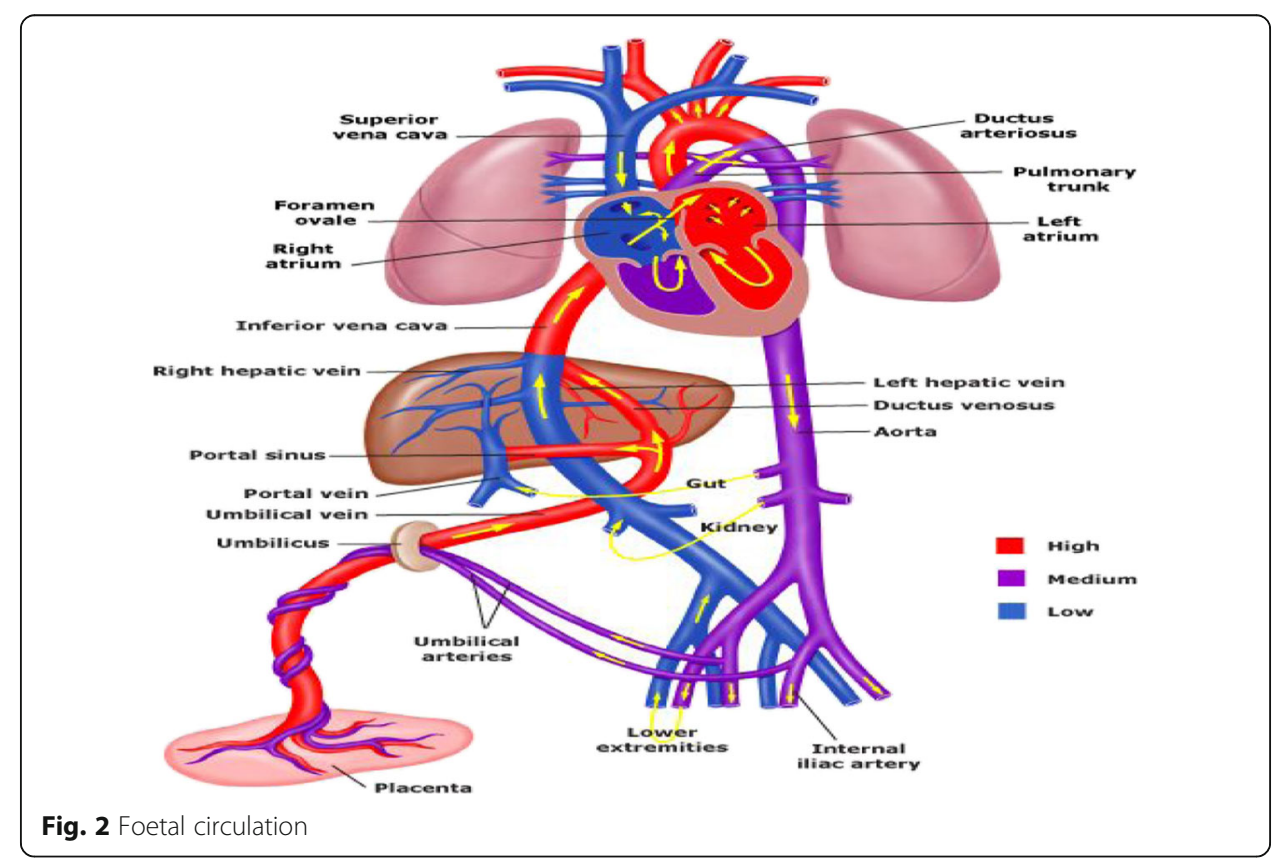


D2] Foetal circulation differs from adult circulation in a variety of ways to support the unique physiologic needs of a developing foetus. Once there is adequate foetalplacental circulation established, blood transports between foetus and placenta through the umbilical cord containing two umbilical arteries and one umbilical vein. The umbilical arteries carry deoxygenated foetal blood to the placenta for replenishment, and the umbilical vein carries newly oxygenated and nutrient-rich blood back to the foetus. When delivering oxygenated blood throughout the developing foetus, there are unique physiologic needs, supported by specific structures unique to the foetus which facilitate these needs.

Through the medical application, we can mention a new topological model. From it, we can know each vertex in foetal circulation and what are the regions that send and receive the blood by dividing the foetal circulation into groups of vertices and edges and forming the graph on it (Fig. 2) [19]. Also, we can conclude the nano topology and NI $\alpha$-open sets on it. In the graph, we consider the foetal circulation as a graph $G=(V, E)$ by working to divide it into a set of vertices and a set of edges. The vertices represent the regions where the blood flows on it. Also, the edges represent the pathway of blood through the foetal circulation (Fig. 3) [19]. The vertices $v_{1}, v_{2}, v_{3}$ and $v_{4}$ (high oxygen content) represent placenta, umbilical vein, liver and ductus venosus respectively; the vertices $v_{6}, v_{7}, v_{8}, v_{9}, v_{10}, v_{14}, v_{15}$, $v_{16}$ and $v_{17}$ (medium oxygen content) represent right atrium, right ventricle, foramen ovale, pulmonary trunk, lung, ductus arteriosus, aorta, systemic circulation and umbilical arteries respectively. Also, the vertices $v_{5}, v_{11}, v_{12}$ and $v_{13}$ (low oxygen content) represent inferior vena cava, left atrium and left ventricle respectively.

From the previous figures, we can construct the graph of the foetal circulation as shown in Fig. 4. It is easy to generate the nano topology $\tau_{R}$ on it by using the neighbourhood of each vertex.

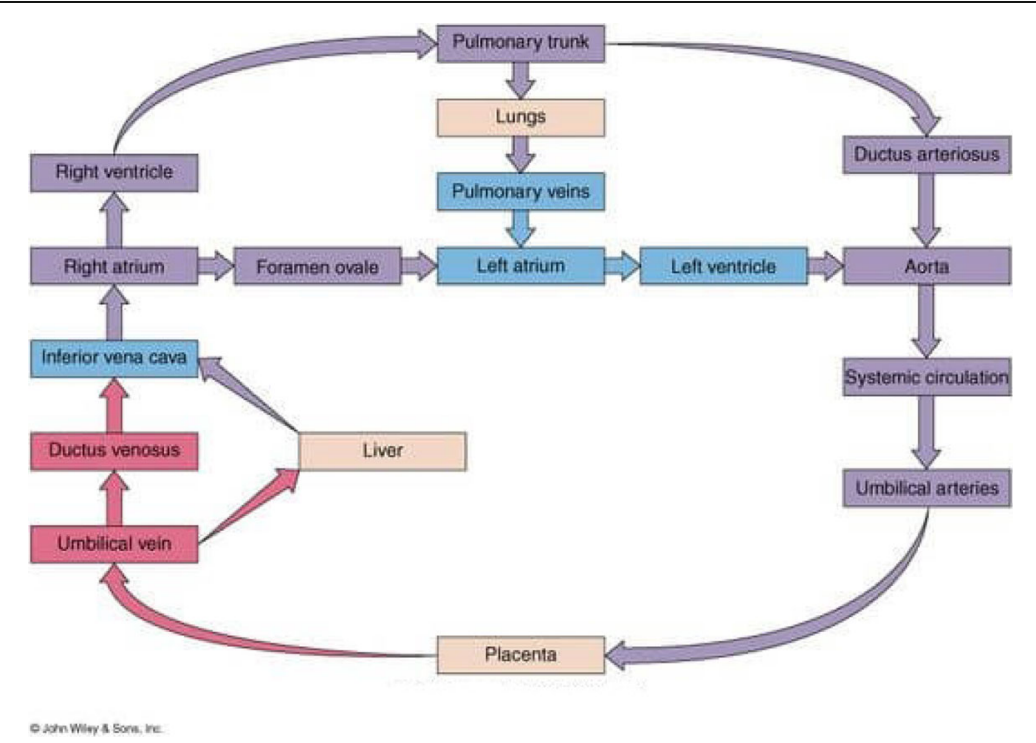

Fig. 3 Foetal circulation step by step 
The neighbourhood of each vertex: $N_{v_{1}}=\left\{v_{1}, v_{2}\right\}, N_{v_{2}}=\left\{v_{2}, v_{3}, v_{4}\right\}, N_{v_{3}}=\left\{v_{3}, v_{5}\right\}, N_{v_{4}}=\left\{v_{4}, v_{5}\right\}$, $N_{v_{5}}=\left\{v_{5}, v_{6}\right\}, N_{v_{6}}=\left\{v_{6}, v_{7}, v_{8}\right\}, N_{v_{7}}=\left\{v_{7}, v_{9}\right\}, N_{v_{8}}=\left\{v_{8}, v_{12}\right\}, N_{v_{9}}=\left\{v_{9}, v_{10}, v_{14}\right\}, N_{v_{10}}=\left\{v_{10}, v_{11}\right\}$, $N_{v_{11}}=\left\{v_{11}, v_{12}\right\}, N_{v_{12}}=\left\{v_{12}, v_{13}\right\}, N_{v_{13}}=\left\{v_{13}, v_{15}\right\}, N_{v_{14}}=\left\{v_{14}, v_{15}\right\}, N_{v_{15}}=\left\{v_{15}, v_{16}\right\}, N_{v_{16}}=\left\{v_{16}, v_{17}\right\}$, $N_{v_{17}}=\left\{v_{1}, v_{17}\right\}$. Take $V(A)=\left\{v_{1}, v_{2}, v_{3}, v_{4}, v_{5}, v_{11}, v_{12}, v_{13}\right\}$ represent the vertices where high and medium oxygen content, then $L(V(A))=\left\{v_{1}, v_{2}, v_{3}, v_{4}, v_{11}, v_{12}\right\}, U(V(A))=\left\{v_{1}, v_{2}, v_{3}, v_{4}, v_{5}, v_{8}, v_{11}, v_{12}, v_{13}, v_{17}\right\}$, therefore $\tau_{R}(A)=\left\{V(G), \emptyset,\left\{v_{5}, v_{8}, v_{13}, v_{17}\right\},\left\{v_{1}, v_{2}, v_{3}, v_{4}, v_{11}, v_{12}\right\},\left\{v_{1}, v_{2}, v_{3}, v_{4}, v_{5}, v_{8}, v_{11}, v_{12}, v_{13}, v_{17}\right\}\right\}$.

Let $I=\left\{\varnothing,\left\{v_{1}\right\}\right\}$, so $N I \alpha O(V(A))=\tau_{R}(V(A)) \cup\{V(\mathbb{A})\}$, where $\{V(\mathbb{A})\} \subset V(G)$ is every set containing the following set $\left\{v_{1}, v_{2}, v_{3}, v_{4}, v_{5}, v_{8}, v_{11}, v_{12}, v_{13}, v_{17}\right\}$. So NIaO $(V(A))=\left\{V(G), \emptyset,\left\{v_{5}, v_{8}\right.\right.$, $\left.v_{13}, v_{17}\right\},\left\{v_{1}, v_{2}, v_{3}, v_{4}, v_{11}, v_{12}\right\},\left\{v_{1}, v_{2}, v_{3}, v_{4}, v_{5}, v_{8}, v_{11}, v_{12}, v_{13}, v_{17}\right\},\left\{v_{1}, v_{2}, v_{3}, v_{4}, v_{5}, v_{6}, v_{8}, v_{11}, v_{12}\right.$, $\left.v_{13}, v_{17}\right\},\left\{v_{1}, v_{2}, v_{3}, v_{4}, v_{5}, v_{7}, v_{8}, v_{11}, v_{12}, v_{13}, v_{17}\right\},\left\{v_{1}, v_{2}, v_{3}, v_{4}, v_{5}, v_{8}, v_{9}, v_{11}, v_{12}, v_{13}, v_{17}\right\},\left\{v_{1}, v_{2}, v_{3}, v_{4}\right.$, $\left.v_{5}, v_{8}, v_{10}, v_{11}, v_{12}, v_{13}, v_{17}\right\},\left\{v_{1}, v_{2}, v_{3}, v_{4}, v_{5}, v_{8}, v_{11}, v_{12}, v_{13}, v_{14}, v_{17}\right\},\left\{v_{1}, v_{2}, v_{3}, v_{4}, v_{5}, v_{8}, v_{11}, v_{12}, v_{13}, v_{15}\right.$, $\left.v_{17}\right\},\left\{v_{1}, v_{2}, v_{3}, v_{4}, v_{5}, v_{8}, v_{11}, v_{12}, v_{13}, v_{16}, v_{17}\right\},\left\{v_{1}, v_{2}, v_{3}, v_{4}, v_{5}, v_{6}, v_{7}, v_{8}, v_{11}, v_{12}, v_{13}, v_{17}\right\},\left\{v_{1}, v_{2}, v_{3}, v_{4}\right.$, $\left.v_{5}, v_{6}, v_{8}, v_{9}, v_{11}, v_{12}, v_{13}, v_{17}\right\}, \ldots\left\{v_{1}, v_{2}, v_{3}, v_{4}, v_{5}, v_{7}, v_{8}, v_{9}, v_{11}, v_{12}, v_{13}, v_{17}\right\},\left\{v_{1}, v_{2}, v_{3}, v_{4}, v_{5}, v_{7}, v_{8}, v_{10}\right.$, $\left.v_{11}, v_{12}, v_{13}, v_{17}\right\}, \ldots,\left\{v_{1}, v_{2}, v_{3}, v_{4}, v_{5}, v_{8}, v_{9}, v_{10}, v_{11}, v_{12}, v_{13}, v_{17}\right\},\left\{v_{1}, v_{2}, v_{3}, v_{4}, v_{5}, v_{8}, v_{9}, v_{11}, v_{12}, v_{13}, v_{14}\right.$, $\left.v_{17}\right\}, \ldots,\left\{v_{1}, v_{2}, v_{3}, v_{4}, v_{5}, v_{8}, v_{10}, v_{11}, v_{12}, v_{13}, v_{14}, v_{17}\right\},\left\{v_{1}, v_{2}, v_{3}, v_{4}, v_{5}, v_{8}, v_{10}, v_{11}, v_{12}, v_{13}, v_{15}, v_{17}\right\}, \ldots,\left\{v_{1}\right.$, $\left.v_{2}, v_{3}, v_{4}, v_{5}, v_{8}, v_{11}, v_{12}, v_{13}, v_{14}, v_{15}, v_{17}\right\},\left\{v_{1}, v_{2}, v_{3}, v_{4}, v_{5}, v_{8}, v_{11}, v_{12}, v_{13}, v_{14}, v_{16}, v_{17}\right\},\left\{v_{1}, v_{2}, v_{3}, v_{4}, v_{5}\right.$, $\left.v_{8}, v_{11}, v_{12}, v_{13}, v_{15}, v_{16}, v_{17}\right\},\left\{v_{1}, v_{2}, v_{3}, v_{4}, v_{5}, v_{6}, v_{7}, v_{8}, v_{9}, v_{11}, v_{12}, v_{13}, v_{17}\right\},\left\{v_{1}, v_{2}, v_{3}, v_{4}, v_{5}, v_{6}, v_{7}, v_{8}\right.$, $\left.v_{10}, v_{11}, v_{12}, v_{13}, v_{17}\right\}, \ldots,\left\{v_{1}, v_{2}, v_{3}, v_{4}, v_{5}, v_{6}, v_{8}, v_{9}, v_{10}, v_{11}, v_{12}, v_{13}, v_{17}\right\},\left\{v_{1}, v_{2}, v_{3}, v_{4}, v_{5}, v_{6}, v_{8}, v_{9}, v_{11}\right.$, $\left.v_{12}, v_{13}, v_{14}, v_{17}\right\}, \ldots,\left\{v_{1}, v_{2}, v_{3}, v_{4}, v_{5}, v_{6}, v_{8}, v_{10}, v_{11}, v_{12}, v_{13}, v_{14}, v_{17}\right\}, \ldots,\left\{v_{1}, v_{2}, v_{3}, v_{4}, v_{5}, v_{6}, v_{8}, v_{11}, v_{12}\right.$, $\left.v_{13}, v_{14}, v_{15}, v_{17}\right\}, \ldots,\left\{v_{1}, v_{2}, v_{3}, v_{4}, v_{5}, v_{6}, v_{8}, v_{11}, v_{12}, v_{13}, v_{15}, v_{16}, v_{17}\right\},\left\{v_{1}, v_{2}, v_{3}, v_{4}, v_{5}, v_{7}, v_{8}, v_{9}, v_{10}, v_{11}\right.$, $\left.v_{12}, v_{13}, v_{17}\right\},\left\{v_{1}, v_{2}, v_{3}, v_{4}, v_{5}, v_{7}, v_{8}, v_{9}, v_{11}, v_{12}, v_{13}, v_{14}, v_{17}\right\}, \ldots,\left\{v_{1}, v_{2}, v_{3}, v_{4}, v_{5}, v_{7}, v_{8}, v_{10}, v_{11}, v_{12}, v_{13}\right.$, $\left.v_{14}, v_{17}\right\},\left\{v_{1}, v_{2}, v_{3}, v_{4}, v_{5}, v_{7}, v_{8}, v_{10}, v_{11}, v_{12}, v_{13}, v_{15}, v_{17}\right\}, \ldots,\left\{v_{1}, v_{2}, v_{3}, v_{4}, v_{5}, v_{7}, v_{8}, v_{11}, v_{12}, v_{13}, v_{14}, v_{15}\right.$, $\left.v_{17}\right\}, \ldots,\left\{v_{1}, v_{2}, v_{3}, v_{4}, v_{5}, v_{7}, v_{8}, v_{11}, v_{12}, v_{13}, v_{15}, v_{16}, v_{17}\right\},\left\{v_{1}, v_{2}, v_{3}, v_{4}, v_{5}, v_{8}, v_{9}, v_{10}, v_{11}, v_{12}, v_{13}, v_{14}, v_{17}\right\}$, $\ldots,\left\{v_{1}, v_{2}, v_{3}, v_{4}, v_{5}, v_{8}, v_{10}, v_{11}, v_{12}, v_{13}, v_{14}, v_{15}, v_{17}\right\}, \ldots,\left\{v_{1}, v_{2}, v_{3}, v_{4}, v_{5}, v_{8}, v_{11}, v_{12}, v_{13}, v_{14}, v_{15}, v_{16}, v_{17}\right\}$ $\left\{v_{1}, v_{2}, v_{3}, v_{4}, v_{5}, v_{6}, v_{7}, v_{8}, v_{9}, v_{10}, v_{11}, v_{12}, v_{13}, v_{17}\right\},\left\{v_{1}, v_{2}, v_{3}, v_{4}, v_{5}, v_{6}, v_{7}, v_{8}, v_{9}, v_{11}, v_{12}, v_{13}, v_{14}, v_{17}\right\}$

$\ldots,\left\{v_{1}, v_{2}, v_{3}, v_{4}, v_{5}, v_{6}, v_{8}, v_{9}, v_{10}, v_{11}, v_{12}, v_{13}, v_{14}, v_{17}\right\},\left\{v_{1}, v_{2}, v_{3}, v_{4}, v_{5}, v_{6}, v_{8}, v_{9}, v_{10}, v_{11}, v_{12}, v_{13}, v_{15}, v_{17}\right\}$, $\ldots,\left\{v_{1}, v_{2}, v_{3}, v_{4}, v_{5}, v_{7}, v_{8}, v_{9}, v_{10}, v_{11}, v_{12}, v_{13}, v_{14}, v_{17}\right\},\left\{v_{1}, v_{2}, v_{3}, v_{4}, v_{5}, v_{7}, v_{8}, v_{9}, v_{10}, v_{11}, v_{12}, v_{13}, v_{15}, v_{17}\right\}$, $\ldots,\left\{v_{1}, v_{2}, v_{3}, v_{4}, v_{5}, v_{8}, v_{9}, v_{10}, v_{11}, v_{12}, v_{13}, v_{14}, v_{15}, v_{17}\right\}, \ldots,\left\{v_{1}, v_{2}, v_{3}, v_{4}, v_{5}, v_{8}, v_{9}, v_{10}, v_{11}, v_{12}, v_{13}, v_{14}, v_{16}\right.$, $\left.v_{17}\right\}, \ldots,\left\{v_{1}, v_{2}, v_{3}, v_{4}, v_{5}, v_{8}, v_{10}, v_{11}, v_{12}, v_{13}, v_{14}, v_{15}, v_{16}, v_{17}\right\},\left\{v_{1}, v_{2}, v_{3}, v_{4}, v_{5}, v_{6}, v_{7}, v_{8}, v_{9}, v_{10}, v_{11}, v_{12}\right.$, $\left.v_{13}, v_{14}, v_{17}\right\},\left\{v_{1}, v_{2}, v_{3}, v_{4}, v_{5}, v_{6}, v_{7}, v_{8}, v_{9}, v_{10}, v_{11}, v_{12}, v_{13}, v_{16}, v_{17}\right\}, \ldots,\left\{v_{1}, v_{2}, v_{3}, v_{4}, v_{5}, v_{7}, v_{8}, v_{9}, v_{10}, v_{11}\right.$, $\left.v_{12}, v_{13}, v_{14}, v_{15}, v_{17}\right\}, \ldots,\left\{v_{1}, v_{2}, v_{3}, v_{4}, v_{5}, v_{8}, v_{9}, v_{10}, v_{11}, v_{12}, v_{13}, v_{14}, v_{15}, v_{16}, v_{17}\right\},\left\{v_{1}, v_{2}, v_{3}, v_{4}, v_{5}, v_{6}, v_{7}\right.$, $\left.\left.v_{8}, v_{9}, v_{10}, v_{11}, v_{12}, v_{13}, v_{14}, v_{15}, v_{17}\right\}, \ldots\right\}$

Take $V(B)=\left\{v_{6}, v_{7}, v_{8}, v_{9}, v_{14}, v_{15}, v_{16}, v_{17}\right\}$ represent the vertices where low oxygen content $\Rightarrow L(V(B))=$ $\left\{v_{6}, v_{7}, v_{8}, v_{14}, v_{15}, v_{16}\right\}, U(V(B))=\left\{v_{5}, v_{6}, v_{7}, v_{8}, v_{9}, v_{13}, v_{14}, v_{15}, v_{16}, v_{17}\right\} \Rightarrow \tau_{\hat{R}}(V(B))=\left\{V(G), \emptyset,\left\{v_{5}, v_{9}\right.\right.$, $\left.\left.v_{13}, v_{17}\right\},\left\{v_{6}, v_{7}, v_{8}, v_{14}, v_{15}, v_{16}\right\},\left\{v_{5}, v_{6}, v_{7}, v_{8}, v_{9}, v_{13}, v_{14}, v_{15}, v_{16}, v_{17}\right\}\right\}$.

Let $J=\left\{\varnothing,\left\{v_{6}\right\}\right\} \Rightarrow N I \alpha O(V(B))=\tau_{R}(V(B)) \cup\{V(\mathbb{B})\}$, where $\{V(\mathbb{B})\} \subset V(G)$ is every set containing the following set $\left\{v_{5}, v_{6}, v_{7}, v_{8}, v_{9}, v_{13}, v_{14}, v_{15}, v_{16}, v_{17}\right\} \Rightarrow \operatorname{NI\alpha } O(V(B))=\left\{V(G), \emptyset,\left\{v_{5}, v_{9}, v_{13}, v_{17}\right\},\left\{v_{6}, v_{7}, v_{8}\right.\right.$, $\left.v_{14}, v_{15}, v_{16}\right\},\left\{v_{5}, v_{6}, v_{7}, v_{8}, v_{9}, v_{13}, v_{14}, v_{15}, v_{16}, v_{17}\right\},\left\{v_{1}, v_{5}, v_{6}, v_{7}, v_{8}, v_{9}, v_{13}, v_{14}, v_{15}, v_{16}, v_{17}\right\},\left\{v_{2}, v_{5}, v_{6}, v_{7}\right.$, $\left.v_{8}, v_{9}, v_{13}, v_{14}, v_{15}, v_{16}, v_{17}\right\},\left\{v_{3}, v_{5}, v_{6}, v_{7}, v_{8}, v_{9}, v_{13}, v_{14}, v_{15}, v_{16}, v_{17}\right\},\left\{v_{4}, v_{5}, v_{6}, v_{7}, v_{8}, v_{9}, v_{13}, v_{14}, v_{15}\right.$, $\left.v_{16}, v_{17}\right\},\left\{v_{5}, v_{6}, v_{7}, v_{8}, v_{9}, v_{10}, v_{13}, v_{14}, v_{15}, v_{16}, v_{17}\right\},\left\{v_{5}, v_{6}, v_{7}, v_{8}, v_{9}, v_{11}, v_{13}, v_{14}, v_{15}, v_{16}, v_{17}\right\},\left\{v_{5}, v_{6}, v_{7}\right.$ $\left.v_{8}, v_{9}, v_{12}, v_{13}, v_{14}, v_{15}, v_{16}, v_{17}\right\},\left\{v_{1}, v_{2}, v_{5}, v_{6}, v_{7}, v_{8}, v_{9}, v_{13}, v_{14}, v_{15}, v_{16}, v_{17}\right\},\left\{v_{1}, v_{3}, v_{5}, v_{6}, v_{7}, v_{8}, v_{9}\right.$, $\left.v_{13}, v_{14}, v_{15}, v_{16}, v_{17}\right\}, \ldots,\left\{v_{1}, v_{3}, v_{5}, v_{6}, v_{7}, v_{8}, v_{9}, v_{13}, v_{14}, v_{15}, v_{16}, v_{17}\right\},\left\{v_{2}, v_{4}, v_{5}, v_{6}, v_{7}, v_{8}, v_{9}, v_{13}, v_{14}, v_{15}\right.$, $\left.v_{16}, v_{17}\right\}, \ldots,\left\{v_{3}, v_{4}, v_{5}, v_{6}, v_{7}, v_{8}, v_{9}, v_{13}, v_{14}, v_{15}, v_{16}, v_{17}\right\},\left\{v_{3}, v_{5}, v_{6}, v_{7}, v_{8}, v_{9}, v_{10}, v_{13}, v_{14}, v_{15}, v_{16}, v_{17}\right\}, \ldots$ $\left\{v_{4}, v_{5}, v_{6}, v_{7}, v_{8}, v_{9}, v_{10}, v_{13}, v_{14}, v_{15}, v_{16}, v_{17}\right\},\left\{v_{4}, v_{5}, v_{6}, v_{7}, v_{8}, v_{9}, v_{11}, v_{13}, v_{14}, v_{15}, v_{16}, v_{17}\right\} \ldots,\left\{v_{5}, v_{6}, v_{7}\right.$, $\left.v_{8}, v_{9}, v_{10}, v_{11}, v_{13}, v_{14}, v_{15}, v_{16}, v_{17}\right\},\left\{v_{5}, v_{6}, v_{7}, v_{8}, v_{9}, v_{10}, v_{12}, v_{13}, v_{14}, v_{15}, v_{16}, v_{17}\right\},\left\{v_{5}, v_{6}, v_{7}, v_{8}, v_{9}, v_{11}\right.$, $\left.v_{12}, v_{13}, v_{14}, v_{15}, v_{16}, v_{17}\right\},\left\{v_{1}, v_{2}, v_{3}, v_{5}, v_{6}, v_{7}, v_{8}, v_{9}, v_{13}, v_{14}, v_{15}, v_{16}, v_{17}\right\},\left\{v_{1}, v_{2}, v_{4}, v_{5}, v_{6}, v_{7}, v_{8}, v_{9}, v_{13}\right.$, $\left.v_{14}, v_{15}, v_{16}, v_{17}\right\}, \ldots,\left\{v_{1}, v_{3}, v_{4}, v_{5}, v_{6}, v_{7}, v_{8}, v_{9}, v_{13}, v_{14}^{1}, v_{15}, v_{16}, v_{17}\right\},\left\{v_{1}, v_{3}, v_{5}, v_{6}, v_{7}, v_{8}, v_{9}, v_{10}, v_{13}, v_{14}\right.$, $\left.v_{15}, v_{16}, v_{17}\right\}, \ldots,\left\{v_{1}, v_{4}, v_{5}, v_{6}, v_{7}, v_{8}, v_{9}, v_{10}, v_{13}, v_{14}, v_{15}, v_{16}, v_{17}\right\},\left\{v_{1}, v_{4}, v_{5}, v_{6}, v_{7}, v_{8}, v_{9}, v_{11}, v_{13}, v_{14}, v_{15}\right.$, $\left.v_{16}, v_{17}\right\}, \ldots,\left\{v_{1}, v_{5}, v_{6}, v_{7}, v_{8}, v_{9}, v_{10}, v_{11}, v_{13}, v_{14}, v_{15}, v_{16}, v_{17}\right\},\left\{v_{1}, v_{5}, v_{6}, v_{7}, v_{8}, v_{9}, v_{16}, v_{17}\right\},\left\{v_{1}, v_{5}, v_{6}, v_{7}\right.$, $\left.v_{8}, v_{9}, v_{11}, v_{12}, v_{13}, v_{14}, v_{15}, v_{16}, v_{17}\right\},\left\{v_{2}, v_{3}, v_{4}, v_{5}, v_{6}, v_{7}, v_{8}, v_{9}, v_{13}, v_{14}, v_{15}, v_{16}, v_{17}\right\},\left\{v_{2}, v_{3}, v_{5}, v_{6}, v_{7}, v_{8}\right.$, $\left.v_{9}, v_{10}, v_{13}, v_{14}, v_{15}, v_{16}, v_{17}\right\}, \ldots,\left\{v_{2}, v_{4}, v_{5}, v_{6}, v_{7}, v_{8}, v_{9}, v_{10}, v_{13}, v_{14}, v_{15}, v_{16}, v_{17}\right\},\left\{v_{2}, v_{4}, v_{5}, v_{6}, v_{7}, v_{8}, v_{9}\right.$ $\left.v_{11}, v_{13}, v_{14}, v_{15}, v_{16}, v_{17}\right\}, \ldots,\left\{v_{2}, v_{5}, v_{6}, v_{7}, v_{8}, v_{9}, v_{10}, v_{11}, v_{13}, v_{14}, v_{15}, v_{16}, v_{17}\right\},\left\{v_{2}, v_{5}, v_{6}, v_{7}, v_{8}, v_{9}\right.$, $\left.v_{10}, v_{12}, v_{13}, v_{14}, v_{15}, v_{16}, v_{17}\right\},\left\{v_{2}, v_{5}, v_{6}, v_{7}, v_{8}, v_{9}, v_{11}, v_{12}, v_{13}, v_{14}, v_{15}, v_{16}, v_{17}\right\},\left\{v_{3}, v_{4}, v_{5}, v_{6}, v_{7}, v_{8}, v_{9}\right.$, $\left.v_{10}, v_{13}, v_{14}, v_{15}, v_{16}, v_{17}\right\},\left\{v_{3}, v_{4}, v_{5}, v_{6}, v_{7}, v_{8}, v_{9}, v_{11}, v_{13}, v_{14}, v_{15}, v_{16}, v_{17}\right\}, \ldots,\left\{v_{3}, v_{5}, v_{6}, v_{7}, v_{8}, v_{9}, v_{10}, v_{11}\right.$ $\left.v_{13}, v_{14}, v_{15}, v_{16}, v_{17}\right\},\left\{v_{3}, v_{5}, v_{6}, v_{7}, v_{8}, v_{9}, v_{10}, v_{12}, v_{13}, v_{14}, v_{15}, v_{16}, v_{17}\right\},\left\{v_{3}, v_{5}, v_{6}, v_{7}, v_{8}, v_{9}, v_{11}, v_{12}, v_{13}\right.$, $\left.v_{14}, v_{15}, v_{16}, v_{17}\right\},\left\{v_{4}, v_{5}, v_{6}, v_{7}, v_{8}, v_{9}, v_{10}, v_{11}, v_{13}, v_{14}, v_{15}, v_{16}, v_{17}\right\},\left\{v_{4}, v_{5}, v_{6}, v_{7}, v_{8}, v_{9}, v_{11}, v_{12}, v_{13}, v_{14}\right.$, $\left.v_{15}, v_{16}, v_{17}\right\},\left\{v_{5}, v_{6}, v_{7}, v_{8}, v_{9}, v_{10}, v_{11}, v_{12}, v_{13}, v_{14}, v_{15}, v_{16}, v_{17}\right\},\left\{v_{1}, v_{2}, v_{3}, v_{4}, v_{5}, v_{6}, v_{7}, v_{8}, v_{9}, v_{13}, v_{14}\right.$, $\left.v_{15}, v_{16}, v_{17}\right\},\left\{v_{1}, v_{2}, v_{3}, v_{5}, v_{6}, v_{7}, v_{8}, v_{9}, v_{10}, v_{13}, v_{14}, v_{15}, v_{16}, v_{17}\right\}, \ldots,\left\{v_{1}, v_{3}, v_{4}, v_{5}, v_{6}, v_{7}, v_{8}, v_{9}, v_{10}, v_{13}\right.$, $\left.v_{14}, v_{15}, v_{16}, v_{17}\right\},\left\{v_{1}, v_{3}, v_{4}, v_{5}, v_{6}, v_{7}, v_{8}, v_{9}, v_{11}, v_{13}, v_{14}, v_{15}, v_{16}, v_{17}\right\}, \ldots,\left\{v_{1}, v_{4}, v_{5}, v_{6}, v_{7}, v_{8}, v_{9}\right.$, $\left.v_{10}, v_{11}, v_{13}, v_{14}, v_{15}, v_{16}, v_{17}\right\},\left\{v_{1}, v_{4}, v_{5}, v_{6}, v_{7}, v_{8}, v_{9}, v_{10}\right.$ $\left.v_{12}, v_{13}, v_{14}, v_{15}, v_{16}, v_{17}\right\},\left\{v_{1}, v_{4}, v_{5}, v_{6}, v_{7}, v_{8}, v_{9}, v_{11}, v_{12}\right.$, $\left.v_{13}, v_{14}, v_{15}, v_{16}, v_{17}\right\},\left\{v_{1}, v_{5}, v_{6}, v_{7}, v_{8}, v_{9}, v_{10}, v_{11}, v_{12}, v_{13}, v_{14}, v_{15}, v_{16}, v_{17}\right\},\left\{v_{2}, v_{3}, v_{4}, v_{5}, v_{6}, v_{7}, v_{8}, v_{9}, v_{10}\right.$, $\left.v_{13}, v_{14}, v_{15}, v_{16}, v_{17}\right\},\left\{v_{2}, v_{3}, v_{4}, v_{5}, v_{6}, v_{7}, v_{8}, v_{9}, v_{11}, v_{13}, v_{14}, v_{15}, v_{16}, v_{17}\right\}, \ldots,\left\{v_{2}, v_{4}, v_{5}, v_{6}, v_{7}, v_{8}, v_{9}, v_{10}\right.$,

$\left.v_{11}, v_{13}, v_{14}, v_{15}, v_{16}, v_{17}\right\}, \ldots,\left\{v_{3}, v_{4}, v_{5}, v_{6}, v_{7}, v_{8}, v_{9}, v_{10}, v_{11}, v_{13}, v_{14}, v_{15}, v_{16}, v_{17}\right\}, \ldots,\left\{v_{4}, v_{5}, v_{6}, v_{7}, v_{8}, v_{9}\right.$ $\left.v_{10}, v_{11}, v_{12}, v_{13}, v_{14}, v_{15}, v_{16}, v_{17}\right\},\left\{v_{1}, v_{2}, v_{3}, v_{4}, v_{5}, v_{6}, v_{7}, v_{8}, v_{9}, v_{10}, v_{13}, v_{14}, v_{15}, v_{16}, v_{17}\right\},\left\{v_{1}, v_{2}, v_{3}, v_{4}, v_{5}\right.$, $\left.v_{6}, v_{7}, v_{8}, v_{9}, v_{11}, v_{13}, v_{14}, v_{15}, v_{16}, v_{17}\right\}, \ldots,\left\{v_{2}, v_{3}, v_{4}, v_{5}, v_{6}, v_{7}, v_{8}, v_{9}, v_{10}, v_{11}, v_{13}, v_{14}, v_{15}, v_{16}, v_{17}\right\}, \ldots,\left\{v_{3}\right.$, $\left.v_{4}, v_{5}, v_{6}, v_{7}, v_{8}, v_{9}, v_{10}, v_{11}, v_{12}, v_{13}, v_{14}, v_{15}, v_{16}, v_{17}\right\},\left\{v_{1}, v_{2}, v_{3}, v_{4}, v_{5}, v_{6}, v_{7}, v_{8}, v_{9}, v_{10}, v_{11}, v_{13}, v_{14}, v_{15}\right.$, $\left.\left.v_{16}, v_{17}\right\}, \ldots\right\}$

Define a function $f:\left(V(G), \tau_{R}(V(A)), I\right) \rightarrow\left(V(G), \tau_{\dot{R}}(V(B)), J\right)$, s.t $f\left(v_{1}\right)=\left\{v_{6}\right\}, f\left(v_{2}\right)=\left\{v_{7}\right\}, f\left(v_{3}\right)=$ $\left\{v_{9}\right\}, f\left(v_{4}\right)=\left\{v_{14}\right\}, f\left(v_{5}\right)=\left\{v_{5}\right\}, f\left(v_{6}\right)=\left\{v_{1}\right\}, f\left(v_{7}\right)=\left\{v_{2}\right\}, f\left(v_{8}\right)=\left\{v_{8}\right\}, f\left(v_{9}\right)=\left\{v_{3}\right\}, f\left(v_{10}\right)=\left\{v_{10}\right\}$, $f\left(v_{11}\right)=\left\{v_{15}\right\}, f\left(v_{12}\right)=\left\{v_{16}\right\}, f\left(v_{13}\right)=\left\{v_{13}\right\}, f\left(v_{14}\right)=\left\{v_{4}\right\}, f\left(v_{15}\right)=\left\{v_{11}\right\}, f\left(v_{16}\right)=\left\{v_{12}\right\}$ and $f\left(v_{17}\right)=$ $\left\{v_{17}\right\}$. This function is nano-continuous, NI $\alpha$-continuous and NI -irresolute continuous but it is not strongly $N I \alpha$-continuous for $\left\{v_{1}, v_{5}, v_{6}, v_{7}, v_{8}, v_{9}, v_{13}, v_{14}, v_{15}, v_{16}, v_{17}\right\} \in N I \alpha O(V(B))$, but $f^{-1}\left(\left\{v_{1}, v_{5}, v_{6}, v_{7}, v_{8}, v_{9}\right.\right.$, $\left.\left.v_{13}, v_{14}, v_{15}, v_{16}, v_{17}\right\}\right)=\left\{v_{1}, v_{2}, v_{3}, v_{4}, v_{5}, v_{6}, v_{8}, v_{11}, v_{12}, v_{13}, v_{17}\right\} \notin \tau_{R}(V(A))$ 


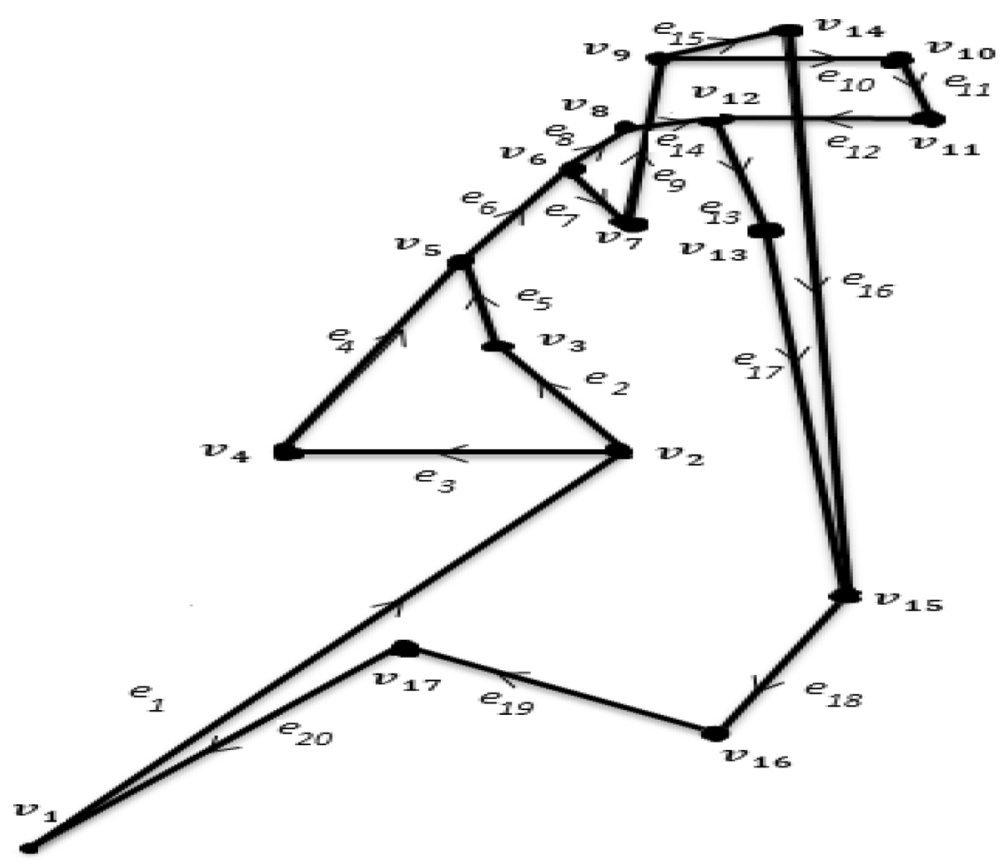

Fig. 4 Graph of foetal circulation

Define a function $f:\left(V(G), \tau_{R}(V(A)), I\right) \rightarrow\left(V(G), \tau_{\hat{R}}(V(B)), J\right)$, such that $f\left(v_{1}\right)=\left\{v_{6}\right\}$,

$$
\begin{aligned}
f\left(v_{2}\right) & =\left\{v_{7}\right\}, f\left(v_{3}\right)=\left\{v_{9}\right\}, f\left(v_{4}\right)=\left\{v_{14}\right\}, f\left(v_{5}\right)=\left\{v_{5}\right\}, f\left(v_{6}\right)=\left\{v_{1}\right\}, f\left(v_{7}\right) \\
& =\left\{v_{2}\right\}, f\left(v_{8}\right)=\left\{v_{8}\right\}, \\
f\left(v_{9}\right) & =\left\{v_{3}\right\}, f\left(v_{10}\right)=\left\{v_{10}\right\}, f\left(v_{11}\right)=\left\{v_{15}\right\}, f\left(v_{12}\right)=\left\{v_{16}\right\}, f\left(v_{13}\right)=\left\{v_{13}\right\}, f\left(v_{14}\right) \\
& =\left\{v_{4}\right\},
\end{aligned}
$$

$f\left(v_{15}\right)=\left\{v_{11}\right\}, f\left(v_{16}\right)=\left\{v_{12}\right\}$ and $f\left(v_{17}\right)=\left\{v_{17}\right\}$. This function is nano-continuous, NI $\alpha$ continuous and NI $\alpha$-irresolute continuous, but it is not strongly NI $\alpha$-continuous for $\left\{v_{1}, v_{5}, v_{6}, v_{7}, v_{8}, v_{9}, v_{13}, v_{14}, v_{15}, v_{16}, v_{17}\right\} \in N I \alpha O(V(B))$, but $f^{-1}\left(\left\{v_{1}, v_{5}, v_{6}, v_{7}, v_{8}, v_{9}, v_{13}, v_{14}\right.\right.$, $\left.\left.v_{15}, v_{16}, v_{17}\right\}\right)=\left\{v_{1}, v_{2}, v_{3}, v_{4}, v_{5}, v_{6}, v_{8}, v_{11}, v_{12}, v_{13}, v_{17}\right\} \notin \tau_{R}(V(A))$.

Also, this function is nano-open, $N I \alpha$-open and $N I \alpha$-almost open, but it is not strongly NI $\alpha$-open for $\left\{v_{1}, v_{2}, v_{3}, v_{4}, v_{5}, v_{6}, v_{8}, v_{11}, v_{12}, v_{13}, v_{17}\right\} \in N I \alpha O(V(A))$, but $f\left(\left\{v_{1}, v_{2}\right.\right.$ $\left.\left., v_{3}, v_{4}, v_{5}, v_{6}, v_{8}, v_{11}, v_{12}, v_{13}, v_{17}\right\}\right)=\left\{v_{1}, v_{5}, v_{6}, v_{7}, v_{8}, v_{9}, v_{13}, v_{14}, v_{15}, v_{16}, v_{17}\right\} \notin \tau_{\hat{R}}(V(B))$.

Clearly, this function is bijective; thus, from the previous properties, $f$ is nanohomeomorphism, $N I \alpha$-homeomorphism and NI $\alpha$-irresolute homeomorphism. Finally, by studying one part of this function, say $A$ and by making new results, this function

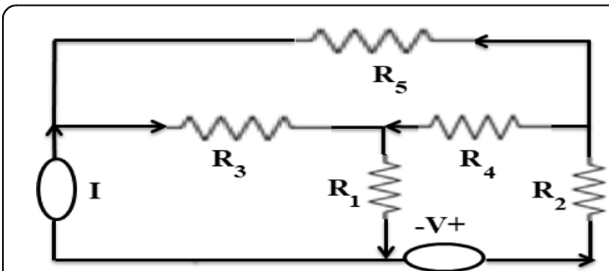

$c_{1}$

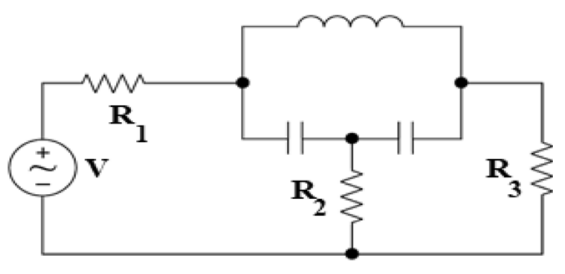

$c_{2}$

Fig. 5 Electrical circuits 
that satisfies $N I \alpha$-irresolute homeomorphism makes the examination of foetal circulation simplest, and by NI $\alpha$-irresolute homeomorphism that preserve all the topological properties of a given space, this new results will be used for the other part of this function, which is $B$. Therefore, there is no need to study all the foetal circulation.

\subsection{Electric circuit}

In this section, we study an application in physics such as an electrical circuit using graphs, nano-topology andNI $\alpha$-open sets. Take two different electrical circuits and transform them into graphs that simply display different graphs. However, we can prove that these circuits have the same electrical properties with ideal nano topology on these graphs.

In Figs. 5 and 6 [20], there are two different electrical circuits $C_{1}$ and $C_{2}$ with two different graphs $G_{1}$ and $G_{2}$, respectively. So, by taking $V(A) \subseteq V\left(G_{1}\right)$ and $V(B) \subseteq V\left(G_{2}\right)$, we can construct a nano topology on them.

The neighborhood of each vertex of $V\left(G_{1}\right): N_{1}=\{1,2\}, N_{2}=\{2,5\}, N_{3}=\{1,2,3\}$, $N_{4}=\{3,4\}$ and $N_{5}=\{1,4,5\}$. So, by taking $V(A)=\{3,4\}$, we get $L(V(A))=\{4\}, U(V(A))=$ $\{3,4,5\}$ and $b(V(A))=\{3,5\}$. Therefore $\tau_{R}(V(A))=\left\{V\left(G_{1}\right), \varnothing,\{4\},\{3,5\},\{3,4,5\}\right\}$. Let $=\{\varnothing,\{1\}\}$. Then $N I \alpha O(V(A))=\left\{V\left(G_{1}\right), \varnothing,\{4\},\{3,5\},\{3,4,5\},\{1,3,4,5\},\{2,3,4,5\}\right\}$.

The neighbourhood of each vertex of $V\left(G_{2}\right): N_{a}=\{a, c\}, N_{b}=\{a, b, e\}, N_{c}=\{c, d, e\}$, $N_{d}=\{b, d\}$ and $N_{e}=\{d, e\}$. So by taking $V(B)=\{a, c\}$, we get $L(V(B))=\{a\}, U(V(B))=\{a$, $b, c\}$ and $b(V(B))=\{b, c\}$. Therefore $\tau_{\hat{R}}(V(B))=\left\{V\left(G_{2}\right), \varnothing,\{a\},\{b, c\},\{a, b, c\}\right\}$. Let $J=\{\varnothing,\{e\}\}$, then $N I \alpha O(V(B))=\left\{V\left(G_{2}\right), \varnothing,\{a\},\{b, c\},\{a, b, c\},\{a, b, c, d\},\{a, b, c, d\}\right\}$.

Define a function $f:\left(V\left(G_{1}\right), \tau_{R}(V(A)), I\right) \rightarrow\left(V\left(G_{2}\right), \tau_{\hat{R}}(V(B)), J\right)$, such that $f(1)=\{e\}$, $f(2)=\{d\}, f(3)=\{c\}, f(4)=\{a\}$ and $f(5)=\{b\}$. This function is nano-continuous, NI $\alpha$-continuous and $N I \alpha$-irresolute continuous, but it is not strongly $N I \alpha$-continuous for $\{a, b$, $c, d\} \in N I \alpha O(V(B))$, but $f^{-1}(\{a, b, c, d\})=\{2,3,4,5\} \notin \tau_{R}(V(A))$. Also, this function is nanoopen, $N I \alpha$-open and $N I \alpha$-almost open, but it is not strongly $N I \alpha$-open for $\{2,3,4,5\} \in$ $N I \alpha O(V(A))$, but $f(\{2,3,4,5\})=\{a, b, c, d\} \notin \tau_{\hat{R}}(V(B))$. Clearly, this function is bijective and from the previous properties $f$ is nano-homeomorphism, NI $\alpha$-homeomorphism and $N I \alpha$-irresolute homeomorphism. Finally, this function which satisfies the NI $\alpha$-irresolute homeomorphism will make the study of the electrical circuit is easier by study one part of this function and made new results on it, then by homeomorphism, these new results can be applied to the other part of this equation.

Another application of NI $\alpha$-irresolute homeomorphism is to prove that two different circuits are identical in their electrical properties. To prove that we define the previous
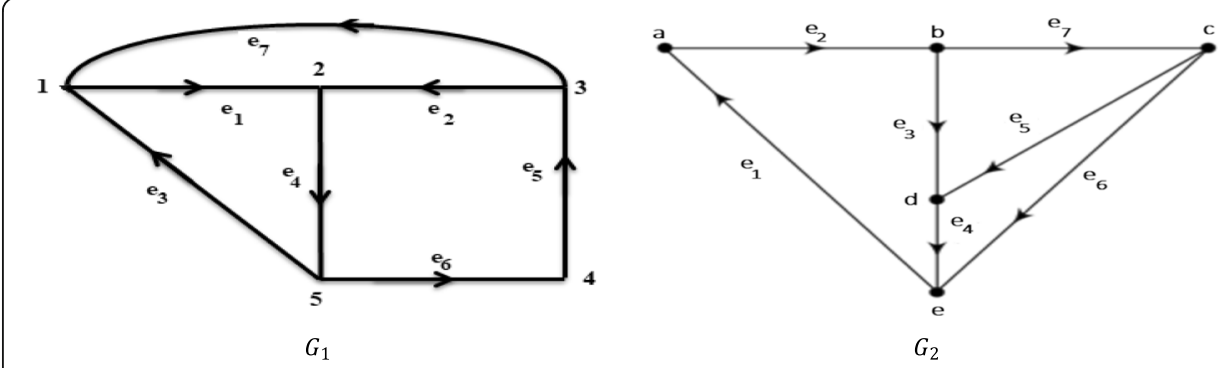

Fig. 6 The corresponding graphs 
function, $f:\left(V\left(G_{1}\right), \tau_{R}(V(A)), I\right) \rightarrow\left(V\left(G_{2}\right), \tau_{\tilde{R}}(V(B)), J\right)$. Clearly, $f$ is an isomorphism. Since $G_{2}$ can be obtained by relabeling the vertices of $G_{1}$, that is, $f$ is a bijection between the vertices of $G_{1}$ and those of $G_{2}$, such that the arcs joining each pair of vertices in $G_{1}$ accepted in both numbers and direction with the arcs joining the corresponding pair of vertices in $G_{2}$.

We also have $f:\left(V\left(G_{1}\right), \tau_{R}(V(A)), I\right) \rightarrow\left(V\left(G_{2}\right), \tau_{\hat{R}}(V(B)), J\right)$ is NI $\alpha$-irresolute homeomorphism for every subgraph $A$ of $G_{1}$, which will be studied in Table 2.

Table 2 Comparison between Nla-irresolute homeomorphisms

\begin{tabular}{|c|c|c|c|}
\hline$V(A)$ & $\mathrm{NlaO}(V(A))$ & $f(V(A))$ & $N / a O(f(V(A)))$ \\
\hline$\varnothing$ & $\left\{V\left(G_{1}\right), \varnothing\right\}$ & $\varnothing$ & $\left\{V\left(G_{2}\right), \varnothing\right\}$ \\
\hline$\{1\}$ & $\left\{V\left(G_{1}\right), \varnothing,\{1,3,5\},\{1,2,3,5\},\{1,3,4,5\}\right\}$ & $\{e\}$ & $\left\{V\left(G_{2}\right), \varnothing,\{b, c, e\},\{b, c, d, e\},\{a, b, c, e\}\right\}$ \\
\hline$\{2\}$ & $\left\{V\left(G_{1}\right), \varnothing,\{1,2,3\},\{1,2,3,4\},\{1,2,3,5\}\right\}$ & $\{d\}$ & $\left\{V\left(G_{2}\right), \varnothing,\{a, d, e\},\{a, c, d, e\},\{b, c, d, e\}\right\}$ \\
\hline$\{3\}$ & $\begin{array}{l}\left\{V\left(G_{1}\right), \varnothing,\{3,4\},\{1,3,4\},\{2,3,4\}\right. \\
\{3,4,5\},\{1,2,3,4\},\{1,3,4,5\},\{2,3,4,5\}\}\end{array}$ & $\{c\}$ & $\begin{array}{l}\left\{V\left(G_{2}\right), \varnothing,\{a, c\},\{a, c, e\},\{a, c, d\},\right. \\
\{a, b, c\},\{a, c, d, e\},\{a, b, c, e\},\{a, b, c, d\}\}\end{array}$ \\
\hline$\{4\}$ & $\begin{array}{l}\left\{V\left(G_{1}\right), \varnothing,\{4,5\},\{1,4,5\},\{2,4,5\}\right. \\
\{3,4,5\},\{1,2,4,5\},\{1,3,4,5\},\{2,3,4,5\}\}\end{array}$ & $\{a\}$ & $\begin{array}{l}\left\{V\left(G_{2}\right), \varnothing,\{a, b\},\{a, b, e\},\{a, b, d\}\right. \\
\{a, b, c\},\{a, b, d, e\},\{a, b, c, e\},\{a, b, c, d\}\}\end{array}$ \\
\hline$\{5\}$ & $\begin{array}{l}\left\{V\left(G_{1}\right), \varnothing,\{2,5\},\{1,2,5\},\{2,3,5\}\right. \\
\{2,4,5\},\{1,2,3,5\},\{1,2,4,5\},\{2,3,4,5\}\}\end{array}$ & $\{b\}$ & $\begin{array}{l}\left\{V\left(G_{2}\right), \varnothing,\{b, d\},\{b, d, e\},\{b, c c d\},\right. \\
\{a, b, d\},\{b, c, d, e\},\{a, b, d, e\},\{a, b, c, d\}\}\end{array}$ \\
\hline$\{1,2\}$ & $\left\{V\left(G_{1}\right), \varnothing,\{1\},\{2,3,5\},\{1,2,3,5\}\right\}$ & $\{d, e\}$ & $\left\{V\left(G_{2}\right), \varnothing,\{e\},\{b, c, d\},\{b, c, d, e\}\right\}$ \\
\hline$\{1,3\}$ & $\left\{V\left(G_{1}\right), \varnothing,\{1,3\},\{4,5\},\{1,3,4,5\}\right\}$ & $\{c, e\}$ & $\left\{V\left(G_{2}\right), \varnothing,\{c, e\},\{a, b\},\{a, b, c, e\}\right\}$ \\
\hline$\{1,4\}$ & $\left\{V\left(G_{1}\right), \varnothing,\{1,3,4,5\}\right\}$ & $\{a, e\}$ & $\left\{V\left(G_{2}\right), \varnothing,\{a, b, c, e\}\right\}$ \\
\hline$\{1,5\}$ & $\left\{V\left(G_{1}\right), \varnothing,\{1,2,3,5\}\right\}$ & $\{b, e\}$ & $\left\{V\left(G_{2}\right), \varnothing,\{b, c, d, e\}\right\}$ \\
\hline$\{2,3\}$ & $\left\{V\left(G_{1}\right), \varnothing,\{1,2,3,4\}\right\}$ & $\{c, d\}$ & $\left\{V\left(G_{2}\right), \varnothing,\{a, c, d, e\}\right\}$ \\
\hline$\{2,4\}$ & $\left\{V\left(G_{1}\right), \varnothing\right\}$ & $\{a, d\}$ & $\left\{V\left(G_{2}\right), \varnothing\right\}$ \\
\hline$\{2,5\}$ & $\left\{V\left(G_{1}\right), \varnothing,\{2\},\{1,3,5\},\{1,2,3,5\}\right\}$ & $\{b, d\}$ & $\left\{V\left(G_{2}\right), \varnothing,\{d\},\{b, c, e\},\{b, c, d, e\}\right\}$ \\
\hline$\{3,4\}$ & $\begin{array}{l}\left\{V\left(G_{1}\right), \varnothing,\{4\},\{3,5\},\{3,4,5\}\right. \\
\{1,3,4,5\},\{2,3,4,5\}\}\end{array}$ & $\{a, c\}$ & $\begin{array}{l}\left\{V\left(G_{2}\right), \varnothing,\{a\},\{b, c\},\{a, b, c\}\right. \\
\{a, b, c, e\},\{a, b, c, d\}\}\end{array}$ \\
\hline$\{3,5\}$ & $\left\{V\left(G_{1}\right), \varnothing,\{2,3,4,5\}\right\}$ & $\{b, c\}$ & $\left\{V\left(G_{2}\right), \varnothing,\{a, b, c, d\}\right\}$ \\
\hline$\{4,5\}$ & $\left\{V\left(G_{1}\right), \varnothing,\{2,4,5\},\{1,2,4,5\},\{2,3,4,5\}\right\}$ & $\{a, b\}$ & $\left\{V\left(G_{2}\right), \varnothing,\{a, b, d\},\{a, b, d, e\},\{a, b, c, d\}\right\}$ \\
\hline$\{1,2,3\}$ & $\left\{V\left(G_{1}\right), \varnothing,\{1,3\},\{2,4,5\}\right\}$ & $\{c, d, e\}$ & $\left\{V\left(G_{2}\right), \varnothing,\{c, e\},\{a, b, d\}\right\}$ \\
\hline$\{1,2,4\}$ & $\left\{V\left(G_{1}\right), \varnothing,\{1\},\{2,3,4,5\}\right\}$ & $\{a, d, e\}$ & $\left\{V\left(G_{2}\right), \varnothing,\{e\},\{a, b, c, d\}\right\}$ \\
\hline$\{1,2,5\}$ & $\left\{V\left(G_{1}\right), \varnothing,\{1,2\},\{3,5\},\{1,2,3,5\}\right\}$ & $\{b, d, e\}$ & $\left\{V\left(G_{2}\right), \varnothing,\{d, e\},\{b, c\},\{b, c, d, e\}\right\}$ \\
\hline$\{1,3,4\}$ & $\left\{V\left(G_{1}\right), \varnothing,\{4\},\{1,3,5\},\{1,3,4,5\}\right\}$ & $\{a, c, e\}$ & $\left\{V\left(G_{2}\right), \varnothing,\{a\},\{b, c, e\},\{a, b, c, e\}\right\}$ \\
\hline$\{1,3,5\}$ & $\left\{V\left(G_{1}\right), \varnothing\right\}$ & $\{b, c, e\}$ & $\left\{V\left(G_{2}\right), \varnothing\right\}$ \\
\hline$\{1,4,5\}$ & $\left\{V\left(G_{1}\right), \varnothing,\{5\},\{1,2,3,4\}\right\}$ & $\{a, b, e\}$ & $\left\{V\left(G_{2}\right), \varnothing,\{b\},\{a, c, d, e\}\right\}$ \\
\hline$\{2,3,4\}$ & $\left\{V\left(G_{1}\right), \varnothing,\{4\},\{4,5\},\{1,2,3,5\}\right\}$ & $\{a, c, d\}$ & $\left\{V\left(G_{2}\right), \varnothing,\{a\},\{a, b\},\{b, c, d, e\}\right\}$ \\
\hline$\{2,3,5\}$ & $\left\{V\left(G_{1}\right), \varnothing,\{2\},\{1,3,4,5\}\right\}$ & $\{b, c, d\}$ & $\left\{V\left(G_{2}\right), \varnothing,\{d\},\{a, b, c, e\}\right\}$ \\
\hline$\{2,4,5\}$ & $\left\{V\left(G_{1}\right), \varnothing,\{2\},\{1,3,4,5\}\right\}$ & $\{a, b, d\}$ & $\left\{V\left(G_{2}\right), \varnothing,\{d\},\{a, b, c, e\}\right\}$ \\
\hline$\{3,4,5\}$ & $\left\{V\left(G_{1}\right), \varnothing,\{2,3,4,5\}\right\}$ & $\{a, b, c\}$ & $\left\{V\left(G_{2}\right), \varnothing,\{a, b, c, d\}\right\}$ \\
\hline$\{1,2,3,4\}$ & $\left\{V\left(G_{1}\right), \varnothing,\{2,5\},\{1,3,4\}\right\}$ & $\{a, c, d, e\}$ & $\left\{V\left(G_{2}\right), \varnothing,\{b, d\},\{a, c, e\}\right\}$ \\
\hline$\{1,2,3,5\}$ & $\left\{V\left(G_{1}\right), \varnothing,\{4,5\},\{1,2,3\}\right\}$ & $\{b, c, d, e\}$ & $\left\{V\left(G_{2}\right), \varnothing,\{a, b\},\{c, d, e\}\right\}$ \\
\hline$\{1,2,4,5\}$ & $\left\{V\left(G_{1}\right), \varnothing,\{3,4\},\{1,2,5\}\right\}$ & $\{a, b, d, e\}$ & $\left\{V\left(G_{2}\right), \varnothing,\{a, c\},\{b, d, e\}\right\}$ \\
\hline$\{1,3,4,5\}$ & $\left\{V\left(G_{1}\right), \varnothing,\{4,5\},\{1,2,3\}\right\}$ & $\{a, b, c, e\}$ & $\left\{V\left(G_{2}\right), \varnothing,\{a, b\},\{c, d, e\}\right\}$ \\
\hline$\{2,3,4,5\}$ & $\left\{V\left(G_{1}\right), \varnothing,\{2,4\},\{1,3,5\}\right\}$ & $\{a, b, c, d\}$ & $\left\{V\left(G_{2}\right), \varnothing,\{a, d\},\{b, c, e\}\right\}$ \\
\hline$V\left(G_{1}\right)$ & $\left\{V\left(G_{1}\right), \varnothing\right\}$ & $V\left(G_{2}\right)$ & $\left\{V\left(G_{2}\right), \varnothing\right\}$ \\
\hline
\end{tabular}


It is clear that from Table 2, the two circuits are NI $\alpha$-irresolute homeomorphism for every subgraph $A$ of $G_{1}$, and using the previous structural equivalence technique we checked that the two circuits are equivalent.

\section{Conclusion}

In this paper, different types of NI $\alpha$-continuous, NI $\alpha$-open, NI $\alpha$-closed and NI $\alpha$-homeomorphism are introduced and studied. Some applications on them are given in some real-life branches such as medicine and physics. We give some examples of electric circuits and study its relationship with graph theory.

\section{Acknowledgements}

Not applicable.

\section{Authors' contributions}

Both authors jointly worked on the results and they read and approved the final manuscript.

Funding

Not applicable.

\section{Availability of data and materials}

The datasets used during the current study are available in the

[D1] https://www.chop.edu/conditions-diseases/blood-circulation-fetus-and-newborn

[D2] https://www.heart.org/en/health-topics/congenital-heart-defects/symptoms\%2D\%2Ddiagnosis-of-congenital-heartdefects/fetal-circulation

[D3] https://www.stanfordchildrens.org/en/topic/default?id=fetal-circulation-90-P01790

The datasets generated during the current study are available from the corresponding author on reasonable request.

\section{Competing interests}

The authors declare that they have no competing interests.

\section{Author details}

${ }^{1}$ Department of Mathematics, Faculty of Science, Tanta University, Tanta 31527, Egypt. ${ }^{2}$ Department of Mathematics, Faculty of Science, Suez Canal University, Ismailia, Egypt.

Received: 7 August 2019 Accepted: 4 June 2020

Published online: 18 August 2020

References

1. Thivagar, M.L., Richard, C.: On nano forms of weakly open sets. International Journal of Mathematics and Statistics Invention. 1(1), 31-37 (2013)

2. Z. Pawlak, Rough sets, Theoretical Aspects of Reasoning About Data, Kluwer Academic Publishers Dordrecht, (1991). https://link.springer.com/article/10.1007/BF01001956

3. Lellis Thivagar, M., Richard, C.: On nano forms of weakly open sets. International Journal of Mathematics and Statistics. 1, 31-37 (2013) https://www.ijmsi.org/Papers/Version.1/E0111031037.pdf

4. Lellis Thivagar, M., Richard, C.: On Nano Continuity. Mathematical Theory and Modelling. 3, 32-37 (2013) http://citeseerx. ist.psu.edu/viewdoc/download?doi=10.1.1.859.3211\&rep=rep1\&type=pdf

5. Lellis Thivagar, M., Manuel, P., Devi, V.S.: A Detection for patent infringement suit via nano topology induced by graph Congent Mathematics. 3, 1161129 (2016) https://doi.org/10.1080/23311835.2016.1161129

6. Nawar, A.S., El-Atik, A.A.: A model of a human heart via graph nano topological spaces. International Journal of Biomathematics. 12(01), 1950006 (2019) https://www.worldscientific.com/doi/pdfplus/10.1142/S1793524519500062

7. Nasef, A.A., El-Atik, A.A.: Some properties on nano topology induced by graphs. AASCIT Journal of Nanoscience. 3(4), 19-23 (2017) http://www.aascit.org/journal/archive2?journalld=970\&paperld=5360

8. L. H. Hsu, C. K. Lin, Graph theory and interconnection networks, CRC Press, (2008).

9. Kuratowski, K.: Topology, Vol I. Academic Press, New York (1933)

10. Vaidyanathaswamy, V.: The localization theory in set topology. Proc. Indian Acad. Sci. 20, 51-61 (1945) https://www.ias. ac.in/article/fulltext/seca/020/01/0051-0061

11. Hayashi, E.: Topologies defined by local properties. Math. Ann. 156, 205-215 (1964). https://link.springer.com/content/ pdf/10.1007/BF01363287.pdf

12. Jankovic, D., Hamlett, T.R.: New topologies from old via ideals. Amer. Math. Monthly. 97, 295-310 (1990) https://doi.org/ 10.1080/00029890.1990.11995593

13. R.L. Newcomb, Topologies which are compact modulo an ideal, Ph.D. Dissertation, Univ. of Cal. at Santa Barbara, (1967).

14. A. A. Nasef, Ideals in general topology, Ph.D., Thesis, Tanta University, (1992).

15. M. E. Abd El-Monsef, A. E. Radwan and A. I. Nasir, Some Generalized forms of compactness in ideal topological spaces, Archives Des Sciences, 66(3) (2013),334- 342. http://www.m-hikari.com/imf/imf-2012/53-56-2012/nasirlMF53-56-2012.pdf

16. A. E. Radwan, A. A. Abd-Elgawad and H. Z. Hassan, On la- connected spaces via ideal, Journal of Advances in Mathematics, 9(9) (2015), 3006- 3014. https://www.researchgate.net/publication/335126206_ON_-CONNECTED_SPACES_VIA_IDEAL 
17. A. E. Radwan, A. A. Abd-Elgawad and H. Z. Hassan, On la- Open Set in Ideal Topological Spaces, Journal of Progressive Research in Mathematics, 6 (2) (2015), 753-760. http://www.scitecresearch.com/journals/index.php/jprm/article/view/451

18. A. M. Kozae, A. A. El-Atik, A. A. Abd-Elgawad and H. Z. Hassan, Ideal expansion for some nano topological structures, Journal of Computer and Mathematical Sciences, 9(11) (2018), 1639-1652. http://compmath-journal.org/detail-d.php?abid=906

19. Stanfordchildrens.org. Default - Stanford Children's Health, (2020) [online]. Available at: https://www.stanfordchildrens. org/en/topic/default?id=fetal-circulation-90-P01790

20. C. Boruah, K. Gogoi and C. Chutia, Analysis of some electrical circuits with the help of graph theory using network equilibrium equations, I. J. of Innovative Research in Science, Engineering and Technology, 6(1) (2017), 944-953. http:// www.ijirset.com/upload/2017/january/63_19_ANALYSIS.pdf

\section{Publisher's Note}

Springer Nature remains neutral with regard to jurisdictional claims in published maps and institutional affiliations.

Submit your manuscript to a SpringerOpen ${ }^{\odot}$ journal and benefit from:

- Convenient online submission

- Rigorous peer review

- Open access: articles freely available online

High visibility within the field

Retaining the copyright to your article 